Claremont Colleges

Scholarship@ Claremont

All HMC Faculty Publications and Research

HMC Faculty Scholarship

$1-1-1994$

\title{
Advection of a Passive Scalar by a Vortex Couple in the Small-diffusion Limit
}

Joseph F. Lingevitch

Northwestern University

Andrew J. Bernoff

Harvey Mudd College

\section{Recommended Citation}

Joseph F. Lingevitch and Andrew J. Bernoff (1994). Advection of a passive scalar by a vortex couple in the small-diffusion limit. Journal of Fluid Mechanics, 270, pp 219-250 doi:10.1017/S0022112094004258

This Article is brought to you for free and open access by the HMC Faculty Scholarship at Scholarship @ Claremont. It has been accepted for inclusion in All HMC Faculty Publications and Research by an authorized administrator of Scholarship @ Claremont. For more information, please contact scholarship@cuc.claremont.edu. 


\title{
Advection of a passive scalar by a vortex couple in the small-diffusion limit
}

\author{
By JOSEPH F. LINGEVITCH AND ANDREW J. BERNOFF \\ Department of Engineering Sciences and Applied Mathematics, Northwestern University, \\ Evanston, IL 60208, USA
}

(Received 11 June 1993 and in revised form 10 December 1993)

We study the advection of a passive scalar by a vortex couple in the small-diffusion (i.e. large Péclet number, $P_{e}$ ) limit. The presence of weak diffusion enhances mixing within the couple and allows the gradual escape of the scalar from the couple into the surrounding flow. An averaging technique is applied to obtain an averaged diffusion equation for the concentration inside the dipole which agrees with earlier results of Rhines \& Young for large times. At the outer edge of the dipole, a diffusive boundary layer of width $O\left(\mathrm{Pe}^{-\frac{1}{2}}\right)$ forms; asymptotic matching to the interior of the dipole yields effective boundary conditions for the averaged diffusion equation. The analysis predicts that first the scalar is homogenized along the streamlines on a timescale $O\left(P e^{\frac{1}{3}}\right)$. The scalar then diffuses across the streamlines on the diffusive timescale; $O(P e)$. Scalar that diffuses into the boundary layer is swept to the rear stagnation point, and a finite proportion is expelled into the exterior flow. Expulsion occurs on the diffusive timescale at a rate governed by the lowest eigenvalue of the averaged diffusion equation for large times. A split-step particle method is developed and used to verify the asymptotic results. Finally, some speculations are made on the viscous decay of the dipole in which the vorticity plays a role analogous to the passive scalar.

\section{Introduction}

The emergence of vortices in two-dimensional turbulent wakes has been observed in experiments and numerical studies (Couder \& Basdevant 1986; McWilliams 1984). A classical example of this is the shedding of a Kármán vortex street in flow around a cylinder at moderate Reynolds numbers (Batchelor 1967; Couder \& Basdevant 1986). Under certain conditions positive and negative vortices can pair to form a vortex couple (Ahlnas, Royer \& George 1987; Couder \& Basdevant 1986). Vortex couples can also form at the head of impulsively started two-dimensional jets and plumes (Conlon \& Lichter 1994; van Heist \& Flór 1989); they are the primary agent for the transport of the injected momentum and vorticity. Unlike an isolated vortex which is advected about by the background fluid flow, an isolated vortex couple propagates in a straight line with constant velocity. This self-propagation property makes vortex couples important structures for transport of passive contaminants (e.g. heat, pollutants) and dynamical quantities (e.g. momentum, vorticity) from a wake or jet into the quiescent background flow.

A canonical example of a vortex couple is a solution of the two-dimensional Euler equations called a Lamb dipole. Figure 1 (a) is the streamfunction of the Lamb dipole viewed in a frame of reference translating with the dipole (Batchelor 1967). The streamlines within the vortex couple are closed and it is surrounded by an irrotational background flow. In this paper we will study the advection and diffusion of a passive 

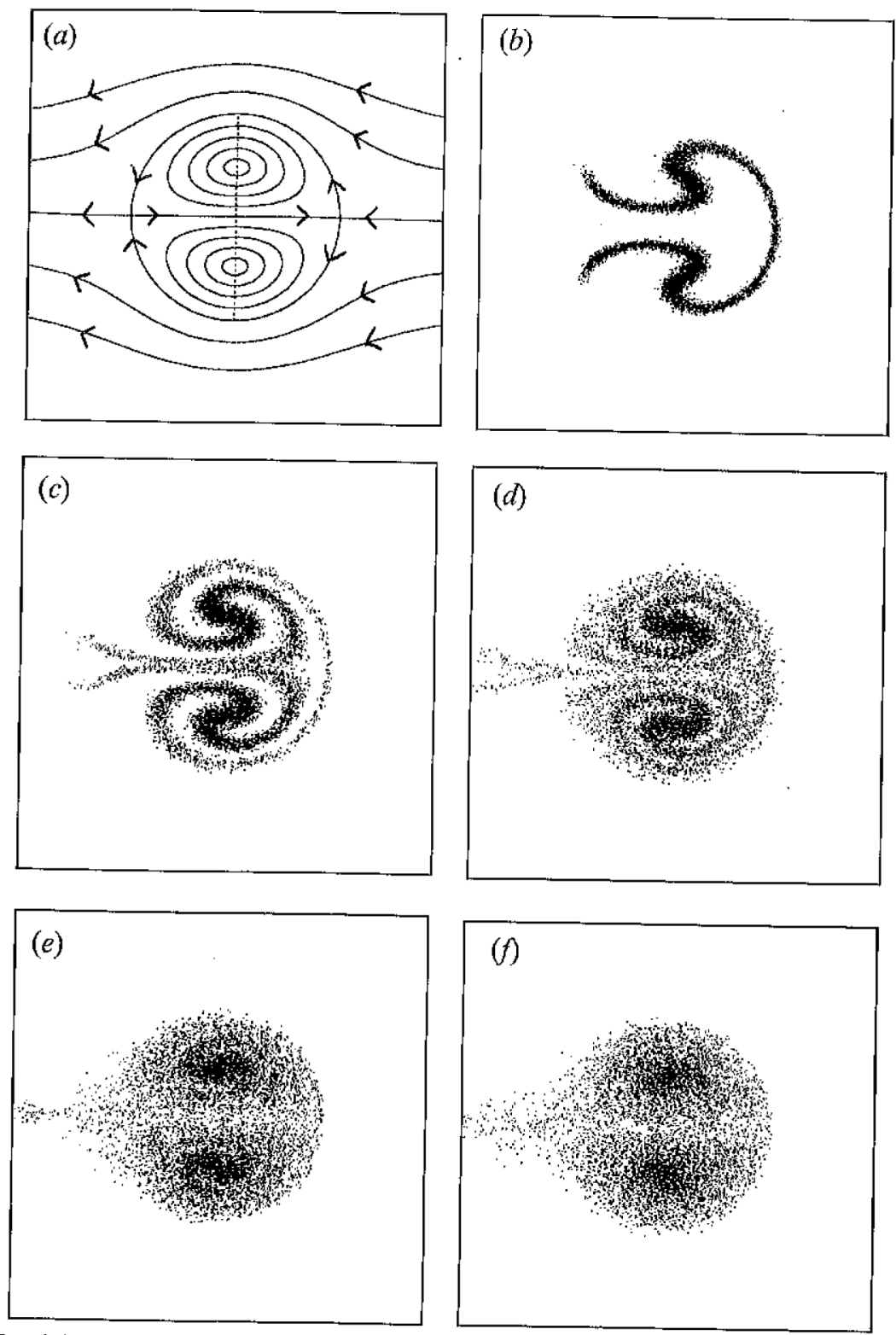

Figure 1. Particle-method simulation of the advection and diffusion of a passive scalar by a Lamb dipole. (a) Streamlines of a Lamb vortex in a co-moving reference frame. The mixing of a line source of passive scalar initially released along the vertical dotted line is considered. $(b-f)$ The numerical solution of the advection-diffusion equation (3.1) at times $t=1,2,3,4,5$ with $P e=1000$. Note that the shear in the dipole causes the scalar to become wrapped in a tight spiral while the diffusion spreads the width of the initial condition. The concentration becomes homogenized along the streamlines and slowly diffuses out of the dipole, being swept towards the rear stagnation point and into the exterior flow.

scalar by a Lamb dipole. We consider the physically relevant case of small scalar diffusion; this weak diffusion can enhance mixing in flows through the interaction of shear and cross-stream diffusion (Rhines \& Young 1983; Taylor 1953).

The mixing of a passive scalar by the Lamb dipole velocity field is illustrated in figure $1(a-f)$ for the case where the diffusive timescale is much longer than the characteristic 
advective turnover time (i.e. large Péclet number $P e$ ). The numerical method is a splitstep particle method where advection along the streamlines of the flow is alternated with diffusion of the particles by a random walk (Appendix A). The density of particles corresponds to the concentration field of the passive scalar. An initial line of passive scalar along the diameter is advected by the velocity field and diffuses (figure $1 a$ ). Owing to shear, the initial condition is wound into a spiral with finer laminations as time progresses (figure $1 b-e$ ). Ultimately cross-stream diffusion acts to smooth out the fine-scale structure produced by the shear (figure $1 f$ ). The combined effect of shear and cross-stream diffusion is to homogenize the passive scalar on the streamlines on a timescale that is much longer than the advective turnover time but much shorter than the diffusive timescale. The scalar will then slowly diffuse across the outer streamline of the couple into the exterior flow, resulting in the slow decay of the scalar concentration within the dipole.

The mixing of a passive-scalar distribution by a two-dimensional flow with closed streamlines for large Péclet numbers was described in the work of Rhines \& Young (1983). They identified two stages of mixing. First, an arbitrary initial concentration is averaged along closed streamlines by a shear-dispersion mechanism. Then the averaged distribution diffuses across streamlines according to a one-dimensional diffusion equation. The mechanism of shear dispersion was first identified by Taylor (1953; see also Young \& Jones 1991) in his derivation of an effective equation for the transport of solute in a tube. This mechanism is responsible for the rapid homogenization of scalar along streamlines in figure 1. In this paper a WKB technique is applied to yield an averaged advection-diffusion equation which describes this homogenization and subsequent diffusion across streamlines for large Péclet numbers.

This averaging technique breaks down at the outer streamline of the Lamb dipole, where the turnover time becomes infinite owing to the presence of stagnation points at the front and the rear of the dipole. Scalar that diffuses across this streamline is swept to the rear stagnation point and into the exterior flow (figure 1). This results in a diffusive boundary layer at the edge of the dipole. The averaged diffusion equation is not valid in this boundary layer where diffusion and advection are comparable effects. Thus a boundary-layer analysis will be applied to describe the escape of scalar from the dipole.

Similar diffusive boundary layers occur in the study of advection-diffusion by an infinite array of Rayleigh-Bénard convection cells (Rosenbluth et al. 1987; Shraiman 1987; Young, Pumir \& Pomeau 1989; Knobloch \& Merryfield 1992). In Rosenbluth et al. (1987) and Shraiman (1987) a steady-state solution is found for an externally applied large-scale concentration gradient. Diffusive boundary layers form between the cells; their net effect is to greatly enhance the diffusion of passive scalar transverse to the roll axes of the convection cells. The steady-state boundary-layer problem is formulated and solved using methods first applied in magnetic dynamo theory (Childress 1979; Childress \& Soward 1989; Perkins \& Zweibel 1987; Soward 1987). In contrast, the decay of the concentration field within the dipole is a non-steady problem that evolves on a slow timescale. The boundary layer must therefore be matched to a non-zero scalar gradient on the interior of the dipole. The method of homogenization has also been applied to the Rayleigh-Bénard problem and to more general singly and doubly periodic flows (Fannjiang \& Papanicolaou 1994; Knobloch \& Merryfield 1992; McCarty \& Horsthemke 1988). The method of homogenization applies averaging over a spatially periodic domain and avoids a detailed boundary-layer analysis. In contrast, for an isolated dipole, the detailed structure of the boundary layer is important in describing the escape of scalar into the outer flow and the homogenization method 
cannot be applied. In Young et al. (1989) non-steady solutions of the Rayleigh-Bénard problem are found for an initial release of scalar in a single cell. An effective leadingorder boundary condition at the edges of the cells is derived and it is shown that the initial diffusive spread of scalar in the convection cells is anomalous and adjusts to Fickian diffusion for large times. Here for the Lamb dipole an effective leading-order boundary condition is derived by a flux balance argument. A detailed boundary-layer analysis then yields the first correction to this effective boundary condition.

The advection-diffusion equation for a passive scalar is the same as the twodimensional Navier-Stokes equation for vorticity with vorticity replacing the concentration of the passive scalar. In the same way that diffusion depletes scalar within the dipole, viscous diffusion will allow for the viscous decay of the dipole. Of course, vorticity is a dynamic quality that is coupled to the velocity field but its transport is closely related to the passive-scalar problem. In high-Reynolds-number flows the viscous dissipation of the vortex couple occurs on a slow timescale thus allowing the couple to propagate large distances (Swaters 1988). The slow escape of vorticity from the dipole will allow for the slow deceleration and distortion of the dipole. This will be the subject of future research. In this limit of viscous diffusion much smaller than scalar diffusion, the strength of the vortex couple will remain essentially constant as the scalar is dispersed (Newton \& Meiburg 1991).

In this paper a quantitative theory for the mixing of an arbitrary initial concentration of passive scalar and its escape rate from the Lamb dipole are developed in the limit of small diffusion. In $\$ 2$ the non-dimensional Lamb-dipole streamfunction and advection-diffusion equation are presented. A WKB averaging method is applied in $\S 3$ to yield an averaged diffusion equation in the region of closed streamlines. It is shown that this averaged diffusion equation describes the rapid homogenization of scalar along streamlines and subsequent expulsion of a large-scale concentration gradient from the dipole on the diffusive timescale. After an initial averaging time of $O\left(P e^{\frac{1}{3}}\right)$ the WKB result reduces to the one-dimensional diffusion equation derived by Rhines \& Young (1983; see also Fowler 1985). At the outer edge of the dipole near the streamline that separates the flow with closed streamlines from the outer irrotational flow, a diffusive boundary layer forms and the averaged diffusion equation breaks down. In $\S 4$, the boundary-layer theory in the vicinity of the outer streamline is developed. The leading-order effective boundary condition for the averaged diffusion equation is shown to be zero concentration at the outer streamline. In $\$ \$ 4.2$ and 4.3 a detailed analysis of the boundary-layer equations leads to an integral equation for the first correction to the boundary layer. The matching condition between the boundary layer and interior concentration field yields the first correction to the effective boundary condition of the averaged diffusion equation. In $\$ 5.1$ the averaged diffusion equation for the Lamb dipole is solved by an eigenfunction expansion. In $\S 5.2$ the eigenfunction solution is compared with a numerical solution of the advection-diffusion equation. It is demonstrated that the asymptotic and numerical predictions of the escape rate of scalar from the dipole are in good agreement. In $\$ 6$ the implications of this analysis for the viscous decay of the Lamb dipole at high Reynolds numbers are discussed. Appendix A describes the split-step particle method used for the numerical solutions. It is shown that the split-step error in the numerical method is $O(\Delta t)^{3}$ per time step. In Appendix $B$ the numerical solution of the boundary-layer integral equation is presented. The numerical method determines the boundary-layer concentration with exponential accuracy. 


\section{The Lamb dipole}

In this section the basic equations governing two-dimensional fluid motion and advection of a passive scalar are discussed. An exact inviscid vortex-couple solution, the Lamb dipole, is described below. We formulate the problem of the advection and small diffusion of a passive scalar by this flow field.

It is convenient to express the Navier-Stokes equations for two-dimensional fluid motion (Batchelor 1967; Saffman 1992) in terms of the advection and diffusion of the scalar vorticity $\omega(x, y)$ :

$$
\omega_{t}+\boldsymbol{u} \cdot \nabla \omega=\frac{1}{R e} \nabla^{2} \omega
$$

where the velocity $\boldsymbol{u}(x, y)$ satisfies incompressibility and can be written in terms of a streamfunction $\psi(x, y)$ :

$$
\boldsymbol{\nabla} \cdot \boldsymbol{u}=0, \quad \boldsymbol{u}=\left(\dot{\psi}_{y},-\psi_{x}\right)
$$

The vorticity is related to the streamfunction by

$$
\nabla^{2} \psi=-\omega
$$

In two dimensions vorticity is a scalar quantity that is advected with the fluid velocity and is diffused by viscosity along vorticity gradients. The strength of the viscous diffusion is specified by the non-dimensional Reynolds number $R e=L U / \nu$, where $L$, $U$ are characteristic length and velocity scales of the flow (specified below) and $\nu$ is the kinematic viscosity. In the limit of large Reynolds number, the viscous diffusion is weak except in those regions where gradients of vorticity are large.

A vortex couple is a pairing of regions of positive and negative vorticity that translates with constant velocity. Consider the inviscid limit of the Navier-Stokes equations in a Galilean reference frame that translates with a vortex couple. In the absence of viscous diffusion $(R e=\infty)$ the vortex couple will satisfy the steady Euler equation:

$$
u \cdot \nabla \omega=0
$$

The gradient of the vorticity is normal to the streamlines, so solutions of the steady Euler equations must satisfy

$$
\omega=F(\psi)
$$

The Lamb dipole (Batchelor 1967) is a particular solution with the vorticity linearly related to the streamfunction inside a circular region of radius $R$ and the flow is irrotational outside this region:

$$
\omega=-\nabla^{2} \psi= \begin{cases}k^{\mathbf{2}} \psi, & r<R \\ 0, & r>R\end{cases}
$$

A dipolar solution to (2.6) is given by

$$
\psi= \begin{cases}A J_{1}(k r) \sin \theta, & r \leqslant R \\ \frac{1}{2} A k R J_{1}^{\prime}(k R)[(r / R)-(R / r)] \sin \theta, & r>R\end{cases}
$$

where $J_{1}$ is the Bessel function of order 1 and $k$ is chosen so that $k R$ is the first zero of $J_{1}$ :

$$
J_{1}(k R)=0 .
$$


The parameter $A$ is the strength of the dipole and determines its propagation velocity. On the boundary of the Lamb dipole $(r=R)$ the streamfunction and its gradient are continuous. The exterior velocity corresponds to irrotational flow past a cylinder with far-field velocity $V=\frac{1}{2} A k R J_{1}^{\prime}(k R)$. In the laboratory reference frame, the Lamb dipole propagates with velocity $V$.

In practice, it is useful to rescale the problem on scales appropriate for the Lamb dipole. If the distances are rescaled with $L=R$ and the velocities with $U=A / R$, this yields the non-dimensional Lamb-dipole streamfunction with no free parameters. The non-dimensional form corresponds to (2.7) with $A=R=1$. At finite Reynolds number the Lamb dipole will decay at a rate dependent upon the rescaled Reynolds number; for large $R e$ it will be argued in $\S 6$ that it decays on an $O(R e)$ timescale.

Similarly to vorticity, a passive scalar is advected with material elements of the flow field; in addition it is also allowed to diffuse. However, as opposed to vorticity, the advecting velocity field is specified independently of the concentration. The governing equation is the two-dimensional advection-diffusion equation:

$$
C_{t}+u \cdot \nabla C=\frac{1}{P e} \nabla^{2} C
$$

where the Péclet number, $P e=L U / \kappa$, is analogous to the Reynolds number with $\kappa$ the diffusion constant for the scalar. Physically, the Péclet number is a ratio of the diffusive to the advective timescales.

Here we consider the case of the transport of a passive-scalar concentration, $C(x, t)$ by the non-dimensional inviscid Lamb dipole velocity field:

$$
\psi= \begin{cases}J_{1}(k r) \sin \theta, & r \leqslant 1 \\ \frac{1}{2} k J_{1}^{\prime}(k)(r-1 / r) \sin \theta, & r>1,\end{cases}
$$

where $k$ is the first zero of $J_{1}$.

In the limit $R e \gg P e \gg 1$ the evolution of the concentration of the passive scalar will occur mich faster than the viscous decay of the vortex couple and the assumption of an inviscid flow field will be a good approximation (Newton \& Meiburg 1991).

\section{Averaged diffusion equation for closed streamlines}

Consider the advection-diffusion equation for the concentration of a passive scalar:

$$
C_{t}+u \cdot \nabla C=\epsilon \nabla^{2} C, \quad u=\left(\psi_{y},-\psi_{x}\right),
$$

where $u(x)$ is a steady two-dimensional flow with closed streamlines, $C(x, t)$ is the concentration, $\not r(x)$ is the streamfunction and $\epsilon$ is the inverse Péclet number $\left(\varepsilon=P e^{-1}\right)$. We consider the case of large Péclet number corresponding to $\epsilon \leqslant 1$. The natural coordinate system for this problem is streamfunction coordinates $(t, x, \theta)$ defined by

$$
\chi(x)=\epsilon^{-\frac{1}{s}} \psi(x), \quad \theta(x)=\Omega(\psi) \int_{C} \frac{\mathrm{d} s}{|\nabla \nabla \gamma|}+\phi(\psi), \quad \Omega(\psi)=2 \pi\left[\oint \frac{\mathrm{d} s}{|\nabla \psi|}\right]^{-1},
$$

where $\chi$ is a scaled streamfunction coordinate and $\theta$ is a $2 \pi$-periodic parameterization of the streamlines. The streamfunction scaling is chosen to balance shear and crossstream diffusion. The integration contour $C$ is a line integral counterclockwise along a streamline from a reference point $x_{0}(\psi)$ to $x$; $\mathrm{d} s$ is a differential arclength. This 
coordinate system will in general be non-orthogonal but the arbitrary initial phase, $\phi(\psi)$ (i.e. the phase at $\left.x_{0}(\psi)\right)$ ), will be determined up to a constant by requiring that $\nabla \psi \cdot \nabla \theta$ averaged over a streamline vanishes (i.e. the coordinate system will be 'orthogonal on the average'). The weighting function $1 /|\nabla \psi|$ in the definition of $\theta(x)$ simplifies the advective time derivative in the streamfunction coordinate system. Physically, the weighting function is related to the spacing between streamlines.

The advection-diffusion equation in this streamfunction coordinate system becomes

$$
C_{t}+\Omega(\psi) C_{\theta}=\epsilon^{\frac{1}{3}}|\nabla \psi /|^{2} C_{\chi \chi}+\epsilon^{\frac{2}{3}}\left(\nabla^{2} \psi C_{\chi}+2 \nabla \theta \cdot \nabla \psi C_{\chi \theta}\right)+\epsilon\left(\nabla^{2} \theta C_{\theta}+|\nabla \theta|^{2} C_{\partial \theta}\right)
$$

The coefficients in the expansion of the Laplacian are $2 \pi$-periodic functions of $\theta$ and are functions of the slow streamfunction coordinate, $e^{\frac{1}{3}} \chi$.

In (3.3) the scalar is advected along the closed streamlines of the flow with angular frequency $\Omega(\psi)$. The frequency $\Omega(\psi)$ is a slowly varying function of $\chi$; thus the scalar will be sheared along the streamlines as it is advected. On the small cross-stream scale, $O\left(\epsilon^{\frac{1}{3}}\right)$, the scalar will be sheared by an order-unity amount along streamlines over times of $O\left(e^{-\frac{1}{3}}\right)$. The sheared scalar becomes wrapped up into thinly spaced layers (see figure 1). The weak cross-stream diffusion smears the width of the layers and homogenizes the concentration in the interstitial regions between the layers. The net result is that the initial concentration is averaged along streamlines on a timescale of $O\left(c^{-\frac{1}{8}}\right)$, when the diffusion width of the layers equals the interstitial spacing, and is known as shear dispersion (Rhines \& Young 1983). The scalar then diffuses across streamlines on the diffusive timescale, $O\left(\epsilon^{-1}\right)$.

The physical interpretation of (3.3) suggests a WKB ansatz for the concentration field (Knobloch \& Merryfield 1992). To leading order, the concentration on particle paths will be constant on the advective timescale. Over longer times diffusion acts to smooth out the small scales produced by shear.

This suggests the ansatz

$$
C(t, \chi, \theta)=C^{0}\left(\xi ; \tau_{n}, \chi\right)+\epsilon^{\frac{1}{3}} C^{1}\left(\xi, \theta ; \tau_{n}, \chi\right)+\ldots,
$$

where

$$
\tau_{n}=\epsilon^{n / 3} t, \quad \xi=\theta-\Omega(\psi) t
$$

The leading-order concentration $C^{0}$ is the mean concentration on the characteristics, constant on the advective timescale and evolving on the slow timescales $\tau_{n}$, due to shear and diffusion. The corrections $\left(C^{n}, n>0\right)$ have period $T=2 \pi / \Omega$ along the characteristics, with zero mean. This ansatz is justified a posteriori by the expansion below.

We define the material derivative,

$$
\frac{\mathrm{D}}{\mathrm{D} t}=\partial_{t}+\Omega(\psi) \partial_{0}
$$

The evolution of the mean concentration $C^{0}$ on the slow timescales is found by requiring that the corrections $\left(C^{1}, C^{2} \ldots\right)$ be $2 \pi$-periodic in $\theta$ or equivalently $T$-periodic on the advective timescale. We now show the details of the WKB averaging theory which results in an averaged diffusion equation for a region of closed streamlines. The $O(1)$ equation describes advection of the initial condition along the streamlines:

$$
\frac{\mathrm{D} C^{0}}{\mathrm{D} t} \equiv C_{t}^{0}+\Omega(\psi) C_{\theta}^{0}=0 .
$$

Note that this requires that $C^{0}$ is a function of $\xi$ and the scaled variables, $\tau_{n}, \chi$. 
At $O\left(6^{\frac{1}{3}}\right)$

$$
\frac{\mathrm{DC}}{\mathrm{D} t}=|\nabla \psi|^{2} C_{\chi \chi}^{0}-C_{\tau_{1}}^{0} \equiv I_{1}(\xi, \theta ; \tau, \chi)
$$

The inhomogeneous term is $2 \pi$-periodic in $\xi$ and $\theta$. There is a solvability condition on the inhomogeneous term for $C^{1}$ to be $T$-periodic on the advective timescale. The solvability condition can be derived by transforming (3.8) to characteristic coordinates $(t, \theta, \chi) \rightarrow(t, \xi, \chi)$. The equation for $C^{1}$ becomes

$$
C_{t}^{1}=I_{1}(\xi, \xi+\Omega t ; \tau, \chi),
$$

where $C^{1}$ will be periodic on the advective timescale if and only if the inhomogeneous term has zero time average over one period, $T$; otherwise it will grow secularly in time. The solvability condition is

$$
\left.\int_{0}^{T} I_{1}\right|_{\xi} \mathrm{d} t=\left.\frac{1}{\Omega} \int_{0}^{2 \pi} I_{1}\right|_{\xi} \mathrm{d} \theta=0
$$

This condition determines the evolution of $C^{0}$ on the $\tau_{1}$ timescale. Defining the average over $\theta$ by an overline,

$$
\bar{f}=\frac{1}{2 \pi} \int_{0}^{2 \pi} f(\theta) \mathrm{d} \theta
$$

the solvability condition for (3.8) becomes

$$
C_{\gamma_{1}}^{0}=\overline{|\nabla \psi|^{2}} C_{\chi \chi^{*}}^{0}
$$

On a timescale of $O\left(\epsilon^{-\frac{1}{6}}\right)$ cross-stream diffusion occurs on a streamfunction scale $O\left(\epsilon^{\frac{1}{3}}\right)$ with diffusion coefficient $\overline{|\nabla \psi /|^{2}}$.

The concentration $C^{1}$ is uniquely determined by (3.8), (3.12) and by requiring that it have zero mean on the characteristics (i.e. $\xi=$ constant):

At $O\left(\epsilon^{\frac{2}{3}}\right)$

$$
\begin{gathered}
C^{1}=(A-\bar{A}) C_{\chi X^{\prime}}^{0} \\
A(\theta, \psi)=\frac{1}{\Omega} \int_{0}^{\theta}\left(|\nabla \psi|^{\prime}-\left.\bar{\nabla} \psi\right|^{2}\right) \mathrm{d} \theta^{\prime} .
\end{gathered}
$$

$$
\begin{aligned}
\frac{\mathrm{D} C^{2}}{\mathrm{D} t} & =|\nabla \psi|^{2} C_{\chi \chi}^{1}+\nabla^{2} \psi C_{\chi}^{0}+2 \nabla \theta \cdot \nabla \psi C_{\chi \theta}^{0}-C_{\tau_{1}}^{1}-C_{\tau_{2}}^{0} \\
& \equiv I_{2}(\xi, \theta ; \tau, \chi),
\end{aligned}
$$

where $C_{\chi \chi}^{1}$ and $C_{\tau_{1}}^{1}$ can be written in terms of derivatives of $C^{0}$ using (3.13a):

$$
\begin{aligned}
& C_{\chi \chi}^{1}=(A-\bar{A}) C_{\chi \chi \chi \chi}^{0}+O\left(\epsilon^{\frac{2}{3}} C_{\chi x}^{0}, \epsilon^{\frac{1}{3}} C_{\chi x \chi}^{0}\right), \\
& C_{\tau_{1}}^{1}=(A-\bar{A})\left(\overline{|\nabla \psi|^{2}} C_{\chi x}^{0}\right)_{\chi x^{*}}
\end{aligned}
$$

The solvability condition, (3.10), applied to $I_{2}$ gives the evolution of $C^{0}$ on the $\tau_{2}$ timescale:

$$
C_{\gamma_{2}}^{0}=\overline{\left(|\nabla \psi|^{2}(A-\bar{A})\right)} C_{\chi \chi \chi X}^{0}+\overline{\nabla^{2} \psi} C_{\chi}^{0}+2 \overline{(\bar{\nabla} \theta \cdot \nabla \psi)} C_{\theta \chi}^{0}-\overline{(A-\bar{A})}\left(\overline{|\nabla \psi|^{2}} C_{\chi x}^{0}\right)_{\chi x}
$$


The function $A-\bar{A}$ is a periodic function of $\theta$ with zero mean so its average is identically zero. Using

$$
A_{0}=\frac{1}{\Omega}\left(|\nabla \psi|^{2}-\overline{|\nabla \psi|^{2}}\right)
$$

a consequence of the definition of $A$ in $(3.13 b)$, yields

$$
\overline{|\nabla \psi|^{2}(A-\bar{A})}=\overline{\left(|\nabla \psi|^{2}-\overline{|\nabla \psi|^{2}}\right)(\overline{A-\bar{A})}}+\overline{|\nabla \psi|^{2}} \overline{(A-\bar{A})}=\Omega \overline{A_{\theta}(A-\bar{A})}=0
$$

By properly choosing the phase $\phi(\psi)$, the coordinate system can be made 'orthogonal on the average':

$$
\phi_{\psi}=-\left[\overline{|\nabla \psi|^{2}}\right]^{-1} \overline{\left(\nabla \psi \cdot\left(\Omega_{\psi} \nabla \psi+\Omega \nabla\right) \int_{x_{0}(\psi)}^{x} \frac{\mathrm{d} s}{|\nabla \psi|}\right)} .
$$

The benefit of this choice is that it eliminates the cross-derivative term in the $O\left(\epsilon^{\frac{2}{3}}\right)$ solvability condition (3.16). This explicit expression for the phase is complicated but mercifully only enters the calculation in computing the diffusion along streamlines. Using (3.18) and (3.19) in (3.16), the evolution of $C^{0}$ on the $\tau_{2}$ timescale simplifies nicely to

$$
C_{\tau_{2}}^{0}=\overline{\nabla^{2} \psi} C_{\chi^{*}}^{0}
$$

The concentration $C^{2}$ is uniquely determined by requiring that it have zero mean on the characteristics; this yields

$$
C^{2}=\left(B_{1}-\overline{B_{1}}\right) C_{\chi x x x}^{0}+\left(B_{2}-\overline{B_{2}}\right) C_{\chi}^{0}+\left(B_{3}-\overline{B_{3}}\right) C_{\chi^{\theta}}^{0} .
$$

The functions $B_{n}$ are $2 \pi$-periodic functions of $\theta$ and the slow streamfunction $\epsilon^{\frac{1}{3}} \chi$.

At $O(\epsilon)$

$$
\begin{aligned}
\frac{\mathrm{D} C^{3}}{\mathrm{D} t}= & |\nabla \psi|^{2} C_{\chi \chi}^{2}+\nabla^{2} \psi C_{\chi}^{1}+2 \nabla \theta \cdot \nabla \psi r C_{\chi \theta}^{1}+\nabla^{2} \theta C_{\theta}^{0}+|\nabla \theta|^{2} C_{\theta \theta}^{0} \\
& -C_{\tau_{3}}^{0}-C_{\tau_{2}}^{1}-C_{\tau_{\alpha}}^{2}+|\nabla \psi|^{2}\left(A_{\psi}-\bar{A}_{\psi}\right) C_{\chi \chi \chi}^{0} \\
\equiv & I_{3}(\xi, \theta ; \tau, \chi) .
\end{aligned}
$$

The solvability condition applied to (3.22) gives the evolution of $C^{0}$ on the $\tau_{\mathrm{a}}$ timescale:

$$
C_{\tau_{3}}^{0}=\overline{|\nabla \theta|^{2}} C_{\theta \theta}^{0}+\overline{\nabla^{2} \theta} C_{\theta}^{0}+O\left(C_{\chi X X}^{0}\right),
$$

corresponding to diffusion along streamlines. The terms in solvability condition (3.23) that are $O\left(C_{x y z}^{0}\right)$ with be $O(\varepsilon)$ when we rescale the streamfunction.

The solvability conditions yield the slow-time evolution of $C^{0}$ in a Lagrangian reference frame. From these we can reconstitute an equation governing the evolution of $C^{0}$ :

$$
\begin{aligned}
\frac{\mathrm{D} C^{0}}{\mathrm{D} t} \equiv & C_{t}^{0}+\Omega(\psi) C_{\theta}^{0}=\epsilon^{\frac{1}{3}} C_{\tau_{1}}^{0}+\epsilon^{\frac{2}{3}} C_{\tau_{2}}^{0}+\epsilon C_{\tau_{3}}^{0}+\ldots \\
= & \left.\epsilon^{\frac{1}{3}} \overline{\nabla \psi} \psi\right|^{2} C_{\chi x}^{0}+\epsilon^{\frac{2}{3}} \overline{\nabla^{2} \psi} C_{\chi}^{0}+\epsilon \mid \overline{\left.\nabla \theta\right|^{2}} C_{\theta \theta}^{0}+\epsilon \overline{\nabla^{2} \psi} C_{\theta}^{0} \\
& +O\left(\epsilon C_{\chi \chi X}^{0} \epsilon^{\frac{4}{8}} C_{\chi \chi}^{0}, \epsilon^{\frac{5}{3}} C_{\chi}^{0}\right) .
\end{aligned}
$$

The error term in (3.24b) arises from higher-order derivatives of concentration with respect to $x$ in the solvability conditions. All of the error terms become $O\left(\epsilon^{2}\right)$ upon rescaling the streamfunction. 
The averaged coefficients in the averaged diffusion equation can be expressed in terms of the circulation, $\zeta(\not r)=\oint|\nabla \psi| \mathrm{d} s$ (where integrals in the counterclockwise direction along streamlines are defined to be positive), and the angular frequency $\Omega(\psi)$ by converting the integrals around streamlines into area integrals (Rhines \& Young 1983). Defining the area integral $Q(\psi)$ of a function $F(x, y)$ as

$$
Q(\psi)=\int_{\mathscr{A}(\psi)} F(x, y) \mathrm{d} A
$$

then the average of $F(x, y)$ around a streamline is related to $Q$ by

$$
\breve{F}=\frac{\Omega(\psi r)}{2 \pi} \frac{\mathrm{d} Q}{\mathrm{~d} \psi}
$$

The integral $Q(\psi)$ can, in some instances, be evaluated in terms of the circulation around the streamline and its derivatives. Using (3.25) and (3.26) the averages of $|\nabla \psi|^{2}$, $\nabla^{2} \psi$ and $\nabla^{2} \theta$ become

$$
\overline{|\nabla \psi|^{2}}=\frac{\Omega(\psi)}{2 \pi} \zeta(\psi), \quad \overline{\nabla^{2} \psi}=\frac{\Omega(\psi r)}{2 \pi} \zeta_{\psi}(\psi), \quad \overline{\nabla^{2} \theta}=0,
$$

where the last expression follows as a consequence of the coordinate system being 'orthogonal on the average'. The expression for the average of $|\nabla \theta|^{2}$ is not simplified by the application of the above identifies. Notice that it only appears in the diffusion along the streamlines. Using (3.27) in (3.24) and rescaling $\psi=\epsilon^{\frac{1}{3}} \chi$, the reconstituted equation for $C^{0}$ becomes

$$
C_{t}^{0}+\Omega(\psi) C_{\theta}^{0}=\epsilon \frac{\Omega(\psi)}{2 \pi}\left(\zeta(\psi) C_{\psi}^{0}\right)_{\psi}+\epsilon|\overline{\nabla \theta} \overline{\mid}|^{2} C_{\theta \theta}^{0}+O\left(\epsilon^{2}\right)
$$

The net result of the averaging is to replace the periodic coefficients of the Laplacian operator in (3.3) with their averages on closed streamlines. Equation (3.28) describes the averaging of an arbitrary initial condition to a state in which concentration is constant along streamlines and the subsequent decay of this homogenized distribution. To show the homogenization of $C^{0}$ we introduce the quantity $I(t)$, a measure of the deviation of the concentration from its average value on streamlines:

where $\bar{C}$ and $\widetilde{C}$ are defined by

$$
I=\frac{1}{2} \int_{a}^{b} \frac{\mathrm{d} \psi}{\Omega} \oint \mathrm{d} \xi \tilde{C}^{2}
$$

$$
\bar{C}=\frac{1}{2 \pi} \int_{0}^{2 \pi} C^{0} \mathrm{~d} \theta, \quad \tilde{C} \equiv C^{0}-\bar{C} .
$$

The quantity $\tilde{C}$ obeys (3.28). Multiplying (3.28) by $\tilde{C}$ and transforming to a rotating coordinate system $(\Psi=\psi, T=t, \xi=\theta-\Omega t)$ yields,

$$
\begin{array}{r}
\frac{1}{2}\left(\tilde{C}^{2}\right)_{T}=\frac{G \Omega}{2 \pi}\left(\frac{1}{2} \zeta\left(\tilde{C}^{2}\right)_{\Psi}\right)_{\Psi}-\frac{1}{2} T\left(\zeta \Omega_{\Psi}\left(\tilde{C}^{2}\right)_{\xi}\right)_{\Psi}-\frac{1}{2} T \zeta \Omega_{\Psi}\left(\tilde{C}^{2}\right)_{\xi \Psi}+\frac{1}{2} T^{2}\left(\Omega_{\Psi}\right)^{2} \zeta\left(\tilde{C}^{2}\right)_{\xi \xi} \\
\left.-\zeta\left(\tilde{C}_{\psi}-\Omega_{\Psi} T \tilde{C}_{\xi}\right)^{2}\right)+\epsilon|\bar{\nabla} \theta|^{2}\left(\frac{1}{2}\left(\tilde{C}^{2}\right)_{\xi \xi}-\left(\tilde{C}_{\xi}\right)^{2}\right) .
\end{array}
$$

Multiplying (3.31) by $1 / \Omega$ and integrating with respect to $\xi$ and $\Psi$ gives an inequality 
for $I_{T}$. Consider an exponentially localized distribution of concentration in the region $a<\psi<b$. Assuming that the timescale for homogenization along streamlines is much faster than that of diffusion across streamlines then the boundary terms from the integration will remain exponentially small during the homogenization. This assumption will be shown to be true in the following. An upper bound for $I_{T}$ satisfies

$$
I_{T} \leqslant-\frac{\epsilon}{2 \pi} \int_{a}^{b} \frac{\mathrm{d} \Psi}{\Omega} \oint \mathrm{d} \xi \zeta \Omega\left(\tilde{C}_{\psi^{\prime}}-\Omega_{\psi} T \tilde{C}_{\xi}\right)^{2} .
$$

The quantity $I(T)$ will be a monotonically decreasing function of time. For large enough times the integrand of (3.32) will be dominated by the term proportional to $T^{2}$; thus $I(T)$ is asymptotically bounded by

$$
I_{T}<-\frac{\epsilon}{2 \pi} \int_{a}^{b} \frac{\mathrm{d} \Psi}{\Omega} \oint \mathrm{d} \xi \zeta \Omega\left(\Omega_{T} T \tilde{C}_{\xi}\right)^{2}
$$

The product of circulation and angular frequency satisfies the inequality

$$
\zeta \Omega \geqslant L u_{m i n} \frac{2 \pi u_{m i n}}{L}=2 \pi u_{m i n}^{2},
$$

where $L$ is the length of the streamline and $u_{m i n}$ is the magnitude of the smallest velocity on the streamline. Using (3.34) and

$$
\oint \mathrm{d} \xi \tilde{C}^{2} \leqslant \oint \mathrm{d} \xi \tilde{C}_{\xi}^{2}
$$

which follows from the fact that $\tilde{C}$ has zero mean, (3.33) implies

$$
\begin{gathered}
I_{T}<-2 \epsilon T^{2} u_{m i n}^{2}\left(\Omega_{\psi}^{2}\right)_{m i n} I, \\
I(T)<I(0) \exp \left(-\epsilon M T^{3}\right) \\
M=\frac{2}{3} u_{m i n}^{2}\left(\Omega_{F}^{2}\right)_{m i n},
\end{gathered}
$$

where $\left(\Omega_{\psi}^{2}\right)_{\min }$ is the minimum value of $\Omega_{\psi}^{2}$ in the region $a \leqslant \psi \leqslant b$. Thus (3.36) shows that an exponentially localized perturbation from the mean concentration will decay exponentially in time on an $O\left(c^{\frac{1}{3}}\right)$ timescale. By the linearity of the advection-diffusion equation we apply the superposition principle to conclude that the leading-order concentration $C^{0}$ is averaged along streamlines on this timescale in regions where $M$ is bounded from below. Notice that the averaging does not occur in regions of zero shear. In the Lamb dipole the shear is bounded away from zero except at the outermost streamline $(\Psi=0)$ where the shear approaches zero like $1 /(\log (\Psi))^{2}$. At the streamline $\Psi=0$ homogenization does not occur because of the vanishing shear. Below we show that the WKB ansatz is invalid at this outermost streamline. Thus the region surrounding this outer streamline will be analysed separately in $\S 4$.

After a time of $O\left(\epsilon^{-\frac{1}{3}}\right)$ the concentration becomes homogenized on the streamlines, $C^{0} \rightarrow \bar{C}$, and (3.28) becomes a one-dimensional diffusion equation for the average concentration on the streamlines:

$$
\bar{C}_{t}=\epsilon \frac{\Omega(\psi r)}{2 \pi}\left(\zeta(\psi r) \bar{C}_{\psi}\right)_{\psi}+O\left(\varepsilon^{2}\right)
$$


This agrees with the one-dimensional diffusion equation obtained by Rhines \& Young (1983). There still remains an $O\left(\epsilon^{\frac{1}{3}}\right)$ periodic modulation of the concentration along streamlines due to the higher-order corrections of the WKB solution but all of the corrections have zero mean $\left(\overline{C^{n}}=0\right.$ for $\left.n>0\right)$ and hence do not contribute to the average concentration along streamlines.

For the WKB averaging technique to be valid it is required that $\epsilon=T_{\text {adv }} / T_{\text {diff }} \ll 1$ : the timescale for diffusion must be much larger than the advective timescale. In terms of dimensional variables this requirement translates to $R^{2} \Omega \gg \kappa$ where $T_{a d v} \sim 1 / \Omega, T_{d i f f} \sim R^{2} / \kappa, R$ is the 'radius' of a closed streamline and $\kappa$ is the diffusion constant. Thus the WKB averaging becomes invalid where $R$ or $\Omega$ go to zero.

For the case of the Lamb dipole, $R$ goes to zero at the centres of the closed streamlines, $\psi=\psi_{m}$, and $\Omega$ approaches zero at the separatrix, $\psi=0$. The breakdown in the averaging at the centres occurs because the advective and diffusive lengthscales are comparable when the radius of the streamlines goes to zero. A local analysis about the centres shows that the streamlines are elliptical. To leading order on small scales near the centres there is a balance between advection and diffusion. In this region the exact solution of the advection-diffusion equation shows that the concentration becomes constant along streamlines on a timescale much faster that the sheardispersion timescale and thus matches with the WKB solution.

The breakdown of the averaging at the separatrix is more severe. In $\S 4$ boundarylayer methods are used to describe the concentration field in the neighbourhood of the separatrix and its escape from the dipole in the boundary-layer region.

\section{Boundary-layer theory}

An averaged diffusion equation, (3.28), for the concentration in a region of closed streamlines was derived in $\S 3$. The concentration is homogenized along the closed streamlines on an $O\left(\epsilon^{\frac{1}{s}}\right)$ timescale, and diffuses across the streamlines on a slower $O(\epsilon)$ timescale. In this section the escape of scalar from the dipole is discussed.

The streamlines for the Lamb dipole change character at $\psi=0$ as shown in figure $1(a)$. On the separatrix, $\psi=0$, there are two stagnation points where the fluid velocity drops to zero. On the interior, there are two regions of closed streamlines and outside the separatrix the streamlines are no longer closed (the outer flow is irrotational). Scalar diffusing across the separatrix may irrecoverably escape from the dipole at the rear stagnation point as it is swept away by the outer flow.

As the closed streamlines approach the separatrix, the period of advection around a streamline becomes infinite owing to the stagnation points. The averaged diffusion equation breaks down as the period of advection approaches the diffusive timescale. In the neighbourhood of the separatrix, a diffusive boundary layer of width $O\left(\epsilon^{\frac{1}{2}}\right)$ forms that connects the concentration in the dipole core with zero concentration in the exterior flow, figure 2. In this diffusive boundary layer the gradients of the concentration are large and diffusive and advective effects are comparable.

In $\$ 4.1$, the neighbourhood of the rear stagnation point is examined. A solution to the advection-diffusion equation valid in this region is divided. It is shown that a finite proportion of the scalar entering the boundary layer is ejected from the rear stagnation point into the outer flow. It is argued heuristically that the leading-order boundary condition on the averaged diffusion equation is vanishing concentration in the boundary layer. In $\$ 4.2$ we derive matched boundary-layer equations for the region near the separatrix. In $\$ 4.3$ we utilize the boundary-layer equations to write an integral equation for the $O\left(\epsilon^{\frac{1}{2}}\right)$ boundary-layer profile. A numerical solution of this integral 


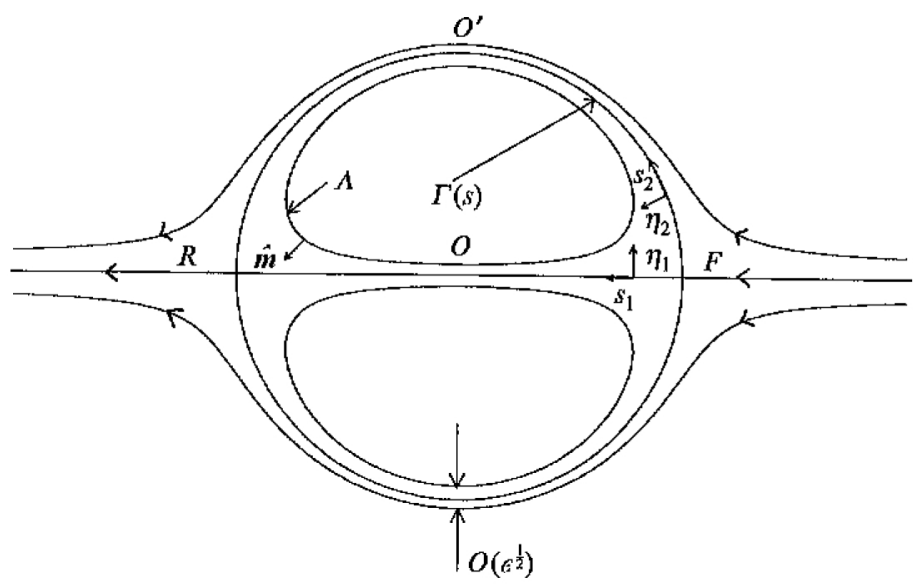

FIGURE 2. Lamb-dipole boundary-layer regions. A diffusive boundary layer of width $O\left(\epsilon^{\frac{1}{2}}\right)$ forms at the outer edge of the dipole and along the centreline. Scalar diffuses into the boundary layer from the interior of the dipole and is flushed downstream at the rear stagnation point $(R)$. Local coordinates $\left(\eta_{1}, s_{1}\right)$ and $\left(\eta_{2}, s_{2}\right)$ are introduced along the streamline $\psi=0$ in the boundary-layer analysis.

equation is found, and matching to the interior flow yields a boundary-condition correction to the averaged diffusion equation of $O\left(\epsilon^{\frac{1}{2}}\right)$.

\subsection{The rear stagnation point}

The rate of escape of scalar from the dipole is constrained by how fast it can escape from the boundary layer into the outer flow. Scalar near the separatrix is swept to the neighbourhood of the rear stagnation point and is either reinjected into the interior of the dipole or escapes into the exterior flow. The exodus of scalar from the dipole at the rear stagnation point can be understood by locally expanding the velocity field in its neighbourhood. In this section the advection-diffusion equation is solved in the neighbourhood of the rear stagnation point, and it is argued heuristically that the concentration falls to zero in the boundary layer.

Consider a coordinate system with its origin at the rear stagnation point. In this vicinity the velocity field can be approximated by $u=(\alpha x,-\alpha y)$, where $\alpha$ determines the strength of the stagnation point flow. An initial point source of scalar at $\left(x_{0}, y_{0}\right)$, within the boundary layer, evolves according to the advection-diffusion equation

$$
C_{t}+\alpha x C_{x}-\alpha y C_{y}=\epsilon \nabla^{2} C+\delta\left(x-x_{0}\right) \delta\left(y-y_{0}\right) \delta(t) .
$$

Rescaling lengths on the boundary-layer scale $X=x / 6^{\frac{1}{2}}$, to balance diffusive and advective effects, yields

$$
C_{t}+\alpha X C_{X}-\alpha Y C_{Y}=\nabla^{2} C+(1 / \epsilon) \delta\left(X-X_{0}\right) \delta\left(Y-Y_{0}\right) \delta(t) .
$$

The weighting of the source term yields an integrated concentration of unity for $t>0$. Equation (4.2) can be solved exactly by transforming to characteristic variables $(x, y, t) \rightarrow(\xi, \eta, T)$ (see Saffman 1992, p. 264; Burgers 1948; and Bernard 1990 for a related treatment):

$$
\xi=\mathrm{e}^{-\alpha t} X, \quad \eta=\mathrm{e}^{\alpha t} Y, \quad T=t .
$$

In these coordinates, (4.2) becomes a diffusion equation with a time-dependent diffusivity:

$$
C_{T}=\mathrm{e}^{-2 \alpha T} C_{\xi \xi}+\mathrm{e}^{2 \alpha T} C_{\eta \eta}+(1 / \epsilon) \delta\left(\xi-X_{0}\right) \delta\left(\eta-Y_{0}\right) \delta(T) .
$$


(a)

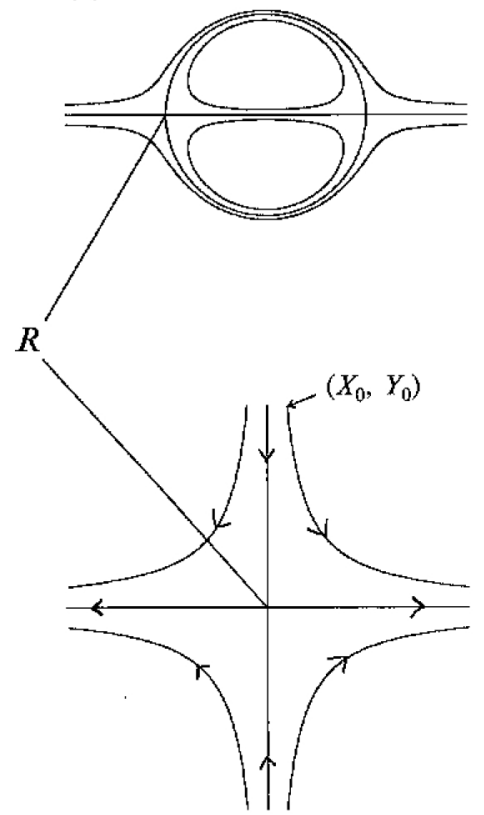

(b)

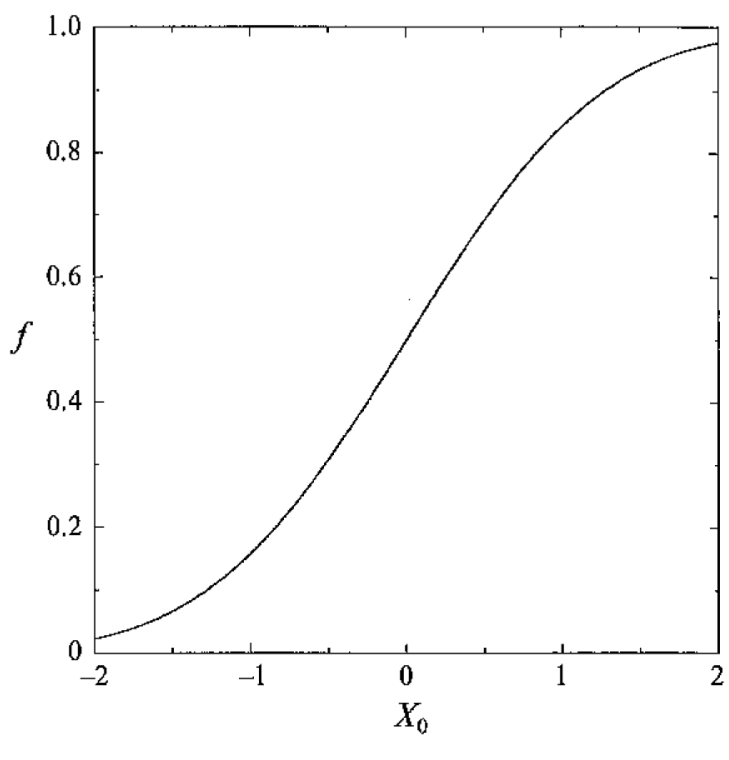

FIGURE 3. Rear-stagnation-point region. (a) In the neighbourhood of the rear stagnation point of the Lamb dipole the leading-order term in the streamfunction is $\psi=\alpha x y$. (b) A fraction $f$ of the scalar released at $\left(X_{0}, Y_{0}\right)$ remains in the right half-plane (interior of the dipole) and the remainder escapes into the left half-plane (outer flow).

Equation (4.4) can be solved with a double Fourier transform in $(\xi, \eta)$. Inverting the transform and converting the solution back to $(X, Y)$ coordinate yields

$$
C(X, Y, T)=\frac{\alpha}{2 \pi\left(\mathrm{e}^{\alpha T}-\mathrm{e}^{-\alpha T}\right)} \exp \left(-\alpha \frac{\left(X \mathrm{e}^{-\alpha T^{\prime}}-X_{0}\right)^{2}}{2\left(1-\mathrm{e}^{-2 \alpha T}\right)}-\alpha \frac{\left(Y \mathrm{e}^{\alpha T^{\prime}}-Y_{0}\right)^{2}}{2\left(\mathrm{e}^{2 \alpha T}-1\right)}\right) .
$$

This solution will be used below to examine the escape of scalar from the dipole near the rear stagnation point.

Consider a point source of concentration in the boundary layer at $\left(X_{0}, Y_{0}\right)$, figure 3 . The fraction of scalar, $f$, that ultimately escapes to the left half-plane can be computed exactly as an integral of (4.5) in the limit as $T \rightarrow \infty$ :

$$
\begin{aligned}
f & =\lim _{T \rightarrow \infty} \int_{-\infty}^{\infty} \mathrm{d} Y \int_{-\infty}^{0} \mathrm{~d} X C(X, Y, T) \\
& =\frac{1}{2}\left(1-\operatorname{erf}\left[X_{0}\left(\frac{1}{2} \alpha\right)^{\frac{1}{2}}\right]\right) .
\end{aligned}
$$

The left half-plane in figure 3 corresponds to the outer flow in the vicinity of the rear stagnation point and the right half-plane corresponds to the interior of the dipole. Consequently, $f$ is the fraction of the scalar that escapes permanently from the dipole. Clearly an order-unity fraction of scalar in the boundary layer escapes at the rear stagnation point.

These results can be interpreted heuristically as follows. The local analysis in the vicinity of the rear stagnation point suggests that the boundary layer expels scalar into the outer flow much faster than scalar can diffuse into the boundary layer from the interior of the dipole. We conclude that the boundary layer adjusts on an order-unity timescale, much faster than the timescale for diffusion in the interior of the dipole. This 
justifies treating the boundary layer of the dipole adiabatically in $\$ 4.2$. The flux out of the boundary layer can be estimated as the integrated concentration in the boundary layer multiplied by the escape rate. Above we argued that the rate of escape of scalar from the boundary layer into the outer flow is order unity, thus $F_{o u t} \propto \epsilon^{\frac{1}{2}} C_{b l}$, where $C_{b l}$ is the boundary-layer concentration. The flux into the boundary layer is supplied from the interior of the dipole and is given by

$$
F_{i n}=\epsilon \oint_{A} \nabla \bar{C} \cdot \hat{m} \mathrm{~d} s \propto \epsilon \bar{C}_{\psi}
$$

where $A$ is an integration contour along a streamline in the matching region between the boundary layer and the interior of the dipole, $\hat{m}$ is a unit normal pointing into the boundary layer from the interior as shown in figure 2 , and $\bar{C}_{\psi} \sim O(1)$ because the gradient of the concentration in the interior of the dipole is of order unity. Equating the fluxes into and out of the boundary layer gives an order of magnitude estimate for the boundary-layer concentration:

$$
C_{b l} \propto e^{\frac{1}{3}} \bar{C}_{y^{\prime}} \ll 1 .
$$

To leading order the concentration in the boundary layer vanishes. In $\$ \$ 4.2$ and 4.3 a boundary condition of the form

$$
\bar{C} \mathcal{L} \epsilon^{\frac{1}{2}} \frac{b}{a} \bar{C}_{\psi}+O(\epsilon) \text { at } \quad y=0
$$

is derived, where $b / a$ is a constant determined in the solution of the leading-order boundary-layer equations. This correction term will yield a boundary layer of thickness $O\left(\epsilon^{\frac{1}{2}}\right)$ at the separatrix of the dipole to smooth the discontinuous concentration gradient present in the leading-order solution.

\subsection{Boundary-layer coordinate transformation}

In this section we formulate the boundary-layer equations for the Lamb dipole in the vicinity of the streamline $\psi=0$. Boundary layers of the type considered here have been studied previously in the context of dynamo theory (Childress 1979; Childress \& Soward 1989; Perkins \& Zweibel 1987; Soward 1987), convection cell transport theory (Rosenbluth et al. 1987; Shraiman 1987; Young et al. 1989) and viscous boundarylayer theory (Harper 1963). In the present case, the boundary-layer solution will yield an $O\left(\epsilon^{\frac{1}{2}}\right)$ correction to the effective boundary condition of the averaged diffusion equation. In the previous work, the boundary-layer equation was transformed into a diffusion equation on the half-plane with zero or constant boundary condition in the far field. In $\$ 4.3$ it is shown that the boundary-layer equations for the Lamb dipole can be formulated as an integral equation for the boundary-layer profile. The boundarylayer concentration must match to a zero concentration outside the dipole and a linearly growing concentration in the interior of the dipole to a match the WKB solution found in $\S 3$.

A local boundary-layer coordinate system illustrated in figure 2 is defined in the neighbourhood of the separatrix, $\psi=0$. Let $x=\Gamma(s)$ be the position vector along the streamline as a function of arclength $s, \hat{n}(s)$ be the unit normal to the curve $\Gamma(s)$, and $\eta$ be a boundary-layer-scaled coordinate along the normal direction. A general position vector and the leading-order terms of the gradient can be computed in $(\eta, s)$ coordinates:

$$
x=\Gamma(s)+\epsilon^{\frac{1}{3}} \eta \hat{n}(s), \quad \nabla=\epsilon^{\frac{1}{2}} \hat{n}(s) \partial_{\eta}+\hat{t}(s) \partial_{s},
$$

where $\hat{t}(s)$ is the unit tangent to $\Gamma(s)$. 
In terms of $(\eta, s)$ coordinates, the leading-order terms in the advection-diffusion equation become

$$
u(s) C_{s}-\eta u^{\prime}(s) C_{\eta}=C_{\eta \eta}+\left(\epsilon C_{s s}\right)+O\left(e^{\frac{1}{2}}\right),
$$

where $u(s)$ is the magnitude of the flow velocity along $\Gamma(s)$. The error term represents higher orders in the Taylor expansion of the velocity and Laplacian. Note also that the time derivative is $O(\epsilon)$ owing to our assumption of an adiabatically adjusting boundary layer. The boundary-layer scaling in (4.10) is valid away from the stagnation points; near a stagnation point, diffusion along the streamlines $\left(\epsilon C_{s s}\right)$ is comparable to diffusion across streamlines as is made clear below.

The boundary layer of the Lamb dipole consists of a piece along the centreline of the dipole $\overline{R O F}$, and a piece along the outer streamline of the dipole at $r=1, \overline{F O^{\prime} R}$ (see figure 2). Equation (4.11) is valid in each of these regions separately and the boundarylayer solutions must be matched at the stagnation points. Here, instead of matching the solutions of the boundary-layer equations we show by a transformation of coordinates that boundary-layer equations from the two regions can be transformed into a single advection-diffusion equation in the neighbourhood of a stagnation point. This transformed equation can then be analysed using the Green's function (4.5) found in $\$ 4.1$.

Here we consider the matching of the boundary-layer equations at the front stagnation point $F$. The matching at the rear stagnation point $R$ proceeds in a similar fashion. Introduce coordinates $\left(\eta_{1}, s_{1}\right)$ for boundary layer $\overline{O F}$ and $\left(\eta_{2}, s_{2}\right)$ for boundary layer $\overline{F O^{\prime}}$ with origin at $F$ as shown in figure 2 . The velocities along the streamline $\psi=0$ in (4.11) are given by $u_{1}\left(s_{1}\right)$ and $u_{2}\left(s_{2}\right)$ for boundary layers $\overline{O F}$ and $\overline{F O^{\prime}}$ respectively. Equation (4.11) for the boundary layers becomes

$$
\begin{aligned}
& \eta_{1} u_{1}^{\prime}\left(s_{1}\right) C_{\eta_{1}}-u_{1}\left(s_{1}\right) C_{s_{1}}=C_{\eta_{1} \eta_{1}}+\epsilon C_{s_{1} s_{1}}+O\left(\epsilon^{\frac{1}{2}}\right) . \\
& \eta_{2} u_{2}^{\prime}\left(s_{2}\right) C_{\eta_{2}}-u_{2}\left(s_{2}\right) C_{s_{2}}=C_{\eta_{2} \eta_{2}}+\epsilon C_{s_{2} s_{2}}+O\left(\epsilon^{\frac{1}{2}}\right) .
\end{aligned}
$$

In $(4,12)$ we retain the streamwise diffusion terms $\epsilon C_{s s}$ because they become important in the overlap region of the boundary layers near $F$. All of the remaining neglected terms are $O\left(c^{\frac{1}{2}}\right)$ throughout the boundary layer. Equations (4.12) can be transformed into a single equation using a change of variable given by

$$
\begin{gathered}
\tilde{\eta}_{1}=\frac{u_{1}\left(s_{1}\right) \eta_{1}}{\alpha \tilde{s}_{1}}, \quad \tilde{s}_{1}=\left[\frac{-2}{\alpha} \int_{s_{1}}^{0} u_{1}\left(s^{\prime}\right) \mathrm{d} s^{\prime}\right]^{\frac{1}{2}}, \\
\tilde{\eta}_{2}=\frac{u_{2}\left(s_{2}\right) \eta_{2}}{\alpha \tilde{s}_{2}}, \quad \tilde{s}_{2}=\left[\frac{2}{\alpha} \int_{0}^{s_{2}} u_{2}\left(s^{\prime}\right) \mathrm{d} s\right]^{\frac{1}{2}},
\end{gathered}
$$

where $\alpha$ is a constant to be specified below. In this twiddled coordinate system equations (4.12) become

$$
\begin{aligned}
& \alpha \tilde{\eta}_{1} C_{\tilde{\eta}_{1}}-\alpha \tilde{s}_{1} C_{\tilde{s}_{1}}=C_{\tilde{\eta}_{1} \tilde{\eta}_{1}}+\left(\epsilon C_{\tilde{s}_{1} \tilde{s}_{1}}\right)+O\left(e^{\frac{1}{2}}\right), \\
& \alpha \tilde{s}_{2} C_{\tilde{s}_{2}}-\alpha \tilde{\eta}_{2} C_{\tilde{\eta}_{2}}=C_{\tilde{\eta}_{2} \tilde{\eta}_{2}}+\left(\epsilon C_{\tilde{s}_{2} \tilde{s}_{2}}\right)+O\left(\epsilon^{\frac{1}{2}}\right),
\end{aligned}
$$

The streamwise diffusion terms $C_{\tilde{\tilde{s}} \tilde{\tilde{\delta}}}$ in (4.14) are negligible except in the coordinate overlap region near $F$. In this overlap region the Taylor expansions of the velocities are $u_{1}\left(s_{1}\right)=\alpha s_{1}+O\left(s_{1}^{2}\right)$ and $u_{2}\left(s_{2}\right)=\alpha s_{2}+O\left(s_{2}^{2}\right)$, where $\alpha$ is the strength of the stagnationpoint flow given by the Taylor expansion of the Lamb-dipole streamfunction at the stagnation point (i.e. $\alpha=-k J_{1}^{\prime}(k)$ ). Substituting these Taylor expansions into (4.13) 
we see that in the coordinate overlap region the change of variables becomes the identity transformation. This suggests that we define a 'composite coordinate system' constructed from the twiddled variables in (4.13):

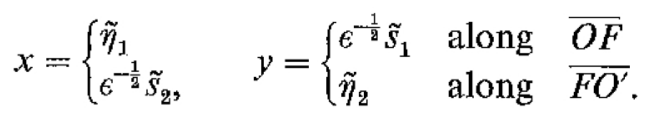

In this coordinate system $(4.14 a, b)$ become a single stagnation-point equation for the concentration in boundary layer $\overline{O F O^{\prime}}$ :

$$
x C_{x}-y C_{y}=C_{x x}+C_{y y}+O\left(\epsilon^{\frac{1}{2}}\right) .
$$

Equation (4.16) is a steady-state advection-diffusion equation in the vicinity of a stagnation point and can be solved using the Green's function (4.5) derived in $\$ 4.1$. The solution of (4.16) for the concentration profile at $O^{\prime}$ due to a unit point source of flux in the boundary layer at $O$ is given asymptotically by

$$
\begin{gathered}
G\left(\Psi, D_{2} ; \Psi_{0}, D_{1}\right) \sim \frac{\exp \left(\frac{-\left(\Psi-\Psi_{0}\right)^{2}}{4\left(D_{1}+D_{2}\right)}\right)}{2\left[\pi\left(D_{1}+D_{2}\right)\right]^{\frac{1}{2}}} F\left(\frac{D_{1} \Psi+D_{2} \Psi_{0}}{2\left[D_{1} D_{2}\left(D_{1}+D_{2}\right)\right]^{\frac{1}{2}}}\right)+O\left(\epsilon^{\frac{1}{2}}\right) \\
F(x)=\frac{1}{2}(1+\operatorname{erf}(x)), \quad \psi=\epsilon^{\frac{1}{2}} \Psi \\
D_{1}=\int_{0}^{s_{O}} u_{1}\left(s^{\prime}\right) \mathrm{d} s^{\prime}, \quad D_{2}=\int_{0}^{s_{o}} u_{2}\left(s^{\prime}\right) \mathrm{d} s^{\prime}
\end{gathered}
$$

where $s_{O}, s_{O^{\prime}}$ are the arclengths from $F$ to $O, O^{\prime}$ respectively along the streamline $\psi=0$. The solution (4.17) is conveniently expressed in terms of the boundary-layer-scaled streamfunction $\Psi$. Similarly the concentration profile at $O$ due to a point source of flux at $O^{\prime}$ is given by $G\left(\Psi^{\prime \prime}, D_{1} ; \Psi, D_{2}\right)$. We use (4.17) in $\S 4.3$ below to construct an integralequation return map for the boundary-layer concentration.

\subsection{Boundary-layer integral equation}

The leading-order boundary layer for the Lamb dipole evolves adiabatically owing to the slowly changing flux into the boundary layer from the interior of the dipole as discussed in $\$ 4.1$. In $\$ 4.2$ we showed that the boundary-layer equations could be transformed into a steady advection-diffusion equation in the neighbourhood of a stagnation point and we found the Green's function solution for this stagnation-point equation. The solution for the boundary layer now becomes an integral equation for the boundary-layer profile. In this section we formulate the integral equation, which can be interpreted as the fixed point of a return map.

The Green's function (4.17) is convoluted with the boundary-layer profile at $O$ to yield the boundary-layer profile at $O^{\prime}$. A similar convolution with the boundary-layer profile at $O^{\prime}$ in turn maps the boundary-layer profile back to $O$ (see figure 2). Periodicity requires that the boundary-layer profile be mapped to itself after one traversal of the boundary layer. This yields the boundary-layer integral equation.

The boundary-layer Green's function (4.17) is essentially a solution to a diffusion equation multiplied by a weighting function that expresses how much of the flux escapes at the stagnation point. In fact the Green's function solution using (4.17) is equivalent to the boundary-layer solution derived by Childress (1979) using a different method. He argues that cross-stream diffusion occurs in the boundary layers between stagnation points but is negligible near the stagnation points owing to the increased separation between streamlines in the vicinity of the stagnation point. The stagnation 
point therefore serves only to advect scalar between boundary layers with negligible diffusion. The advantage of the method presented here is that it allows the formal expansion of the effects at the stagnation point.

The return map for the boundary-layer concentration becomes

$$
\begin{gathered}
C^{\prime}(\Psi)=\int_{0}^{\infty} \mathrm{d} \Psi^{\prime} G_{F^{\prime}}\left(\Psi, \Psi^{\prime}\right) C\left(\Psi^{\prime}\right), \\
C^{\prime \prime}(\Psi)=\int_{-\infty}^{\infty} \mathrm{d} \Psi^{\prime} G_{R}\left(\Psi, \Psi^{\prime}\right) C^{\prime}\left(\Psi^{\prime}\right),
\end{gathered}
$$

where $C, C^{\prime}$ and $C^{\prime \prime}$ are the boundary-layer profiles at $O, O^{\prime}$ and $O$ respectively. The periodicity condition for the boundary layer becomes $C(\Psi)=C^{\prime \prime}(\Psi)$. The kernels $G_{F}$ and $G_{R}$ are constructed from (4.17) so that the concentration satisfies appropriate boundary conditions (to be discussed below).

For the boundary-layer solution at $O$ we impose a symmetry condition that the concentration be an even or odd function of $\Psi$ across the centreline of the dipole, $\overline{F R}$. This will lead to even and odd boundary-layer solutions which will smoothly connect the even and odd eigenfunctions of the reduced diffusion equation. The other boundary condition to be imposed is a requirement that the boundary-layer concentration smoothly match to the WKB solution in the far field. The boundary condition at $O^{\prime}$ is that the concentration should decay exponentially outside the dipole $(\Psi \rightarrow-\infty)$ and the concentration should match the WKB solution on the interior of the dipole.

In the far field $(\Psi \rightarrow \infty)$ the boundary-layer return map (4.18) becomes a convolution of the concentration with a Gaussian and is equivalent to the solution of the diffusion equation:

$$
\bar{C}_{\theta}=\frac{\zeta_{0}}{2 \pi} \bar{C}_{I^{I} T^{\prime},} \quad \Psi \rightarrow \infty
$$

Equation (4.19) is precisely the inner limit of the averaged diffusion equation (3.28) expressed in terms of inner variables and neglecting the time derivative. In the matching regime, the concentration is constant along streamlines and solutions of (4.19) become

$$
\bar{C}=a \Psi+b, \quad \Psi \rightarrow \infty,
$$

where $a$ and $b$ are constants.

It is the interior of the dipole that supplies the concentration flux to support a nontrivial boundary-layer solution. Expressing the matching condition (4.20) in outer variables gives the $O\left(e^{\frac{1}{2}}\right)$ correction to the effective boundary condition of the averaged diffusion equation:

$$
\left.\frac{\bar{C}}{\overline{C_{\psi}}}\right|_{\psi^{\prime}-0}=\epsilon^{\frac{1}{2}} \frac{b}{a}+O(e)
$$

The constants $a$ and $b$ in the matching condition are not independent; their ratio is fixed and they are related by the solution of the boundary layer. Specifying $a$ for instance, fixes the flux into the boundary layer from the interior. Once the flux into the boundary layer is specified, $b$ is determined by the solution of the boundary-layer equation. The boundary-layer solution will complete the specification-of the matching condition.

Boundary-layer integral equations of the type given here have been solved analytically in some instances by the Wiener-Hopf method (Childress 1979; Childress \& Soward 1989; Soward 1987). Thus far we have only obtained a numerical solution 


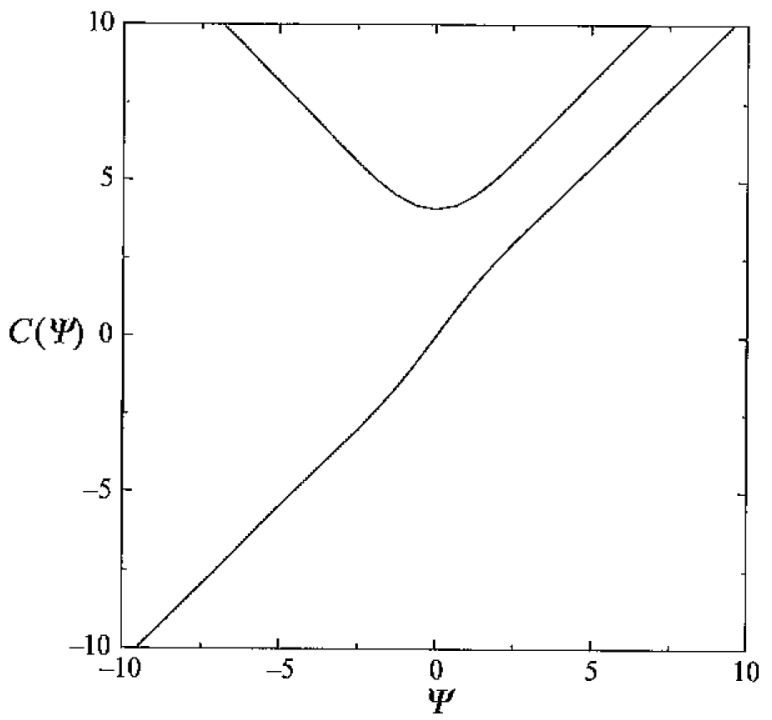

FIGURF 4. Symmetric and antisymmetric boundary-layer solutions. A unit flux of scalar from the interior of the dipole into the boundary layer yields the concentration profiles $C(\Psi)$ at the centre of the Lamb dipole $(O)$ (see figure 2). The concentration in the boundary layer asymptotes to a unit slope in the far field. The intercepts of the asymptotes determine the $O\left(c^{2}\right)$ effective boundary condition of the averaged diffusion equation.

to (4.18) (which is exponentially accurate). The numerical discretization of (4.18) is discussed in Appendix B. Figure 4 shows the symmetric and antisymmetric boundarylayer solutions at $O$. The constant ratio $b / a$ in (4.21) is given by 3.1786375 $(0.4524429)$ for the (anti)symmetric boundary layers.

\section{Decay rate of the averaged concentration}

In this section the numerical solution of the full advection-diffusion equation by a particle method is compared with an eigenfunction solution of the averaged diffusion equation (3.37). In $\$ 5.1$ the leading-order approximation for the smallest eigenvalue is solved exactly. The $O\left(c^{\frac{1}{2}}\right)$ correction to this eigenvalue is computed using the $O\left(\frac{1}{6^{2}}\right)$ correction to the boundary condition (4.21) derived in $\$ 4$. In $\$ 5.2$ the eigenfunction solution is compared to a numerical particle method solution of the advection-diffusion equation for a delta-function initial concentration at the centre of one of the lobes of the Lamb dipole. The eigenfunction and numerical solutions are found to converge in the limit $\epsilon \rightarrow 0$ thus validating our asymptotic analysis.

\subsection{Eigenmodes of the averaged diffusion equation}

In $\S 3$ we elucidated the shear-dispersion mechanism which homogenizes an arbitrary initial concentration in a region of closed streamlines on an $O\left(P e^{\frac{1}{3}}\right)$ timescale. The decay of the averaged concentration is governed by a one-dimensional diffusion equation (3.37) repeated here for convenience:

$$
\bar{C}_{t}=\epsilon \frac{\Omega(\psi)}{2 \pi}\left(\zeta(\psi) \bar{C}_{\psi}\right)_{\psi}
$$

with boundary condition

$$
\bar{C}=\epsilon^{\frac{1}{2}}(b / a) \bar{C}_{\psi} \quad \text { at } \quad \psi=0,
$$


where $b / a$ is a constant determined by the boundary-layer analysis in $\S 4.3$. Here we solve equation (5.1) by an eigenfunction expansion. Owing to the symmetry of the Lamb dipole we consider an eigenfunction expansion in symmetric and antisymmetric eigenmodes satisfying

$$
\phi_{n}^{a}(\psi)=-\phi_{n}^{a}(-\psi), \quad \phi_{n}^{s}(\psi r)=\phi_{n}^{s}(-\psi)
$$

where $\phi^{s}\left(\phi^{a}\right)$ are the symmetric (antisymmetric) eigenfunctions. The eigenfunction expansion for the averaged concentration becomes

$$
\bar{C}(\psi, t)=\sum_{n=0}^{\infty}\left(c_{n} \mathrm{e}^{\mathrm{e} \lambda} \lambda_{n}^{a} t \phi_{n}^{a}(\psi)+d_{n} \mathrm{e}^{\mathrm{e} \lambda_{n}^{s} t} \phi_{n}^{s}(\psi)\right) .
$$

This gives a Sturm-Liouville eigenvalue problem for the decay rates $\lambda_{n}^{a, s}$ and the eigenmodes $\phi_{n}^{a, s}$. In general the antisymmetric and symmetric modes will have different decay rates. All of the decay rates are real and negative and the lowest symmetric eigenvalue, $\lambda_{0}^{s}$, governs the rate of escape of scalar from the dipole. Dropping the (anti)symmetric superscripts to simplify notation, the decay rates and eigenfunctions can be obtained by the perturbation expansion

$$
\begin{aligned}
& \phi_{n}=\phi_{n}^{(0)}+e^{\frac{1}{2}} \phi_{n}^{(1)}+\ldots, \\
& \lambda_{n}=\lambda_{n}^{(0)}+\epsilon^{\frac{1}{2}} \lambda_{n}^{(1)}+\ldots
\end{aligned}
$$

The leading-order problem for the eigenmodes on $\left[0, \psi_{m}\right]$, where $\psi_{m}$ is the maximum value of the streamfunction within the dipole, becomes

$$
\begin{gathered}
L \phi_{n}^{\langle 0\rangle}=\frac{\Omega}{2 \pi}\left(\zeta \phi_{n \psi}^{(0)}\right)_{\Psi}-\lambda_{n}^{(0)} \phi_{n}^{(0)}=0, \\
\phi_{n}^{\langle 0\rangle}=0 \quad \text { at } \quad \psi=0, \quad \lim _{\psi \rightarrow \psi_{m}}\left(\psi-\psi_{m}\right) \phi_{n \psi}^{(0)}=0 .
\end{gathered}
$$

The boundary condition at $\psi=\psi_{m}$ is a boundedness condition on the eigenfunction at $\psi=\psi_{m}$. At leading order the slowest decaying mode $(n=0)$ can be found exactly:

$$
\phi_{0}^{(0)}=A \psi, \quad A=1.72+, \quad \lambda_{0}^{(0)}=-k^{2}=-14.68+,
$$

where $k$ is the first zero of the first Bessel function, $J_{1}$, and the normalization constant, $A$, has been chosen so that $\phi_{0}^{(0)}\left(\psi_{m}\right)=1$. The slowest decaying eigenmode is proportional to the streamfunction at leading order. Note that, the leading-order decay rates for the symmetric and antisymmetric modes are identical. For $n>0$ the eigenmodes and eigenvalues are found numerically by shooting. The first five leadingorder eigenmodes are shown in figure 5.

At $O\left(e^{\frac{1}{2}}\right)$

$$
\begin{aligned}
L \phi_{n}^{(1)} & =\lambda_{n}^{(1)} \phi_{n}^{(0)}, \\
\phi_{n}^{(1)}=(b / a) \phi_{n \psi}^{(0)} \quad \text { at } \quad \psi & =0, \quad \lim _{\psi \rightarrow \psi_{m}}\left(\psi-\psi_{m}\right) \phi_{n \psi}^{(1)}=0 .
\end{aligned}
$$

The homogeneous version of (5.8) has a non-trivial solution so there is a solvability condition on (5.8) in order for a solution of the inhomogeneous problem to exist. The solvability condition will determine the decay rate corrections $\lambda_{n}^{(1)}$ uniquely. The solvability condition is a result of the Fredholm Alternative Theorem. Defining the inner product $\langle u, v\rangle$ :

$$
\langle u, v\rangle=\int_{0}^{\psi m} \frac{\mathrm{d} \psi}{\Omega(\psi)} u(\psi) v(\psi)
$$




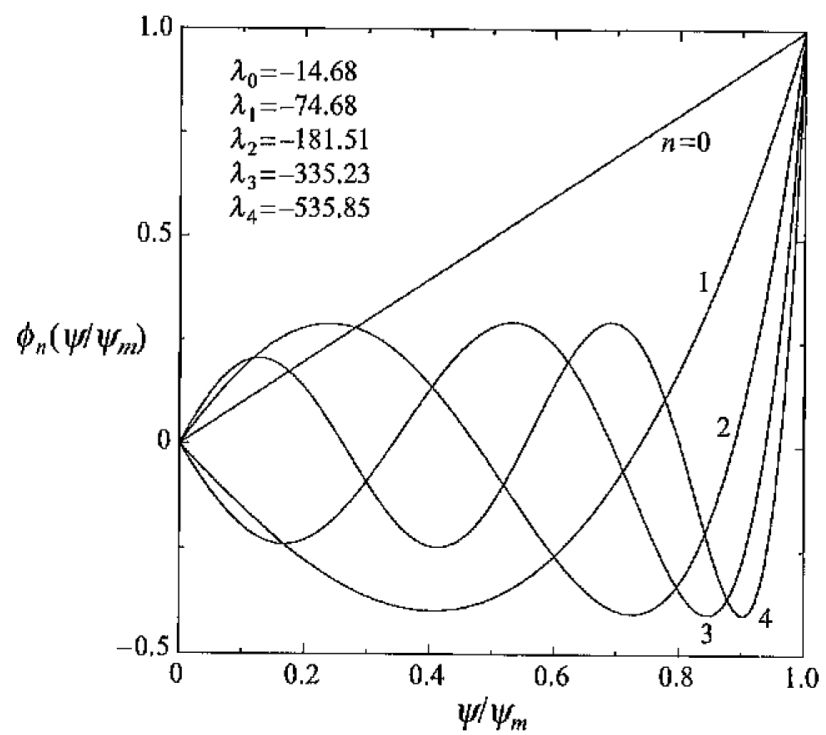

FIGURE 5. First five leading-order eigenmodes of the averaged diffusion equation. The eigenfunctions $\left(\phi_{n 2}\right)$ and eigenvalues $\left(\lambda_{n}\right)$ of the averaged diffusion equation were determined numerically by shooting. Here the streamfunction $\psi$ is divided by its maximum $\psi_{m}$ and the eigenfunctions are normalized to unity at $\psi_{m}$. Note that for $n=0$ the eigenfunction is proportional to the streamfunction.

the solvability condition becomes

$$
\left\langle\phi_{n}^{(0)}, L \phi_{n}^{(1)}\right\rangle=\lambda_{n}^{(1)}\left\langle\phi_{n}^{(0)}, \phi_{n}^{(0)}\right\rangle
$$

Integrating the left-hand side of (5.10) by parts and using boundary condition $(5.8 \mathrm{~b})$ gives an expression for $\lambda_{n}^{(1)}$ :

$$
\lambda_{n}^{(1)}=\frac{(b / a) \zeta_{0}\left(\left.\phi_{n, d}^{(0)}\right|_{\psi^{\prime}=0}\right)^{2}}{2 \pi\left\langle\phi_{n}^{(0)}, \phi_{n}^{(0)}\right\rangle},
$$

where $b / a$ is given by the boundary-layer solution and $\zeta_{0}$ is the circulation about the streamline $\psi=0$. The $O\left(c^{\frac{1}{2}}\right)$ correction to symmetric and antisymmetric decay rates will differ owing to the differing values of ratio $b / a$ for the symmetric and antisymmetric boundary layers (see $\$ 4.3$ ).

The correction to the $n=0$ decay rates can be computed exactly for the Lamb dipole. Using

$$
\left\langle\phi_{0}^{(0)}, \phi_{0}^{(0)}\right\rangle=A^{2}\langle\psi, \psi\rangle=\frac{1}{8} A^{2}\left(J_{1}^{\prime}(k)\right)^{2},\left.\quad \phi_{0 \psi}^{(0)}\right|_{\psi=0}=A,
$$

the $n=0$ decay rates become

$$
\begin{aligned}
& \lambda_{0}^{a}=-14.68+18.72 \epsilon^{\frac{1}{2}}+O(\epsilon), \\
& \lambda_{0}^{s}=-14.68+131.50 \epsilon^{\frac{1}{2}}+O(\epsilon) .
\end{aligned}
$$

With solvability condition (5.11), equations (5.8) are solved for $\phi_{n}^{(1)}$.

\subsection{Comparison of asymptotic and numerical solutions}

The eigenfunctions computed in $\$ 5.1$ are used here to calculate the time evolution of an initial point source of concentration at $\psi=\psi_{m}$ in one of the lobes of the Lamb dipole. Consider a point source at $\psi=\psi_{m}$ normalized so that the integrated concentration within the dipole at $t=0$ is unity:

$$
C(\psi, 0)=\frac{\Omega(\psi)}{2 \pi} \delta\left(\psi-\psi_{m}\right)
$$



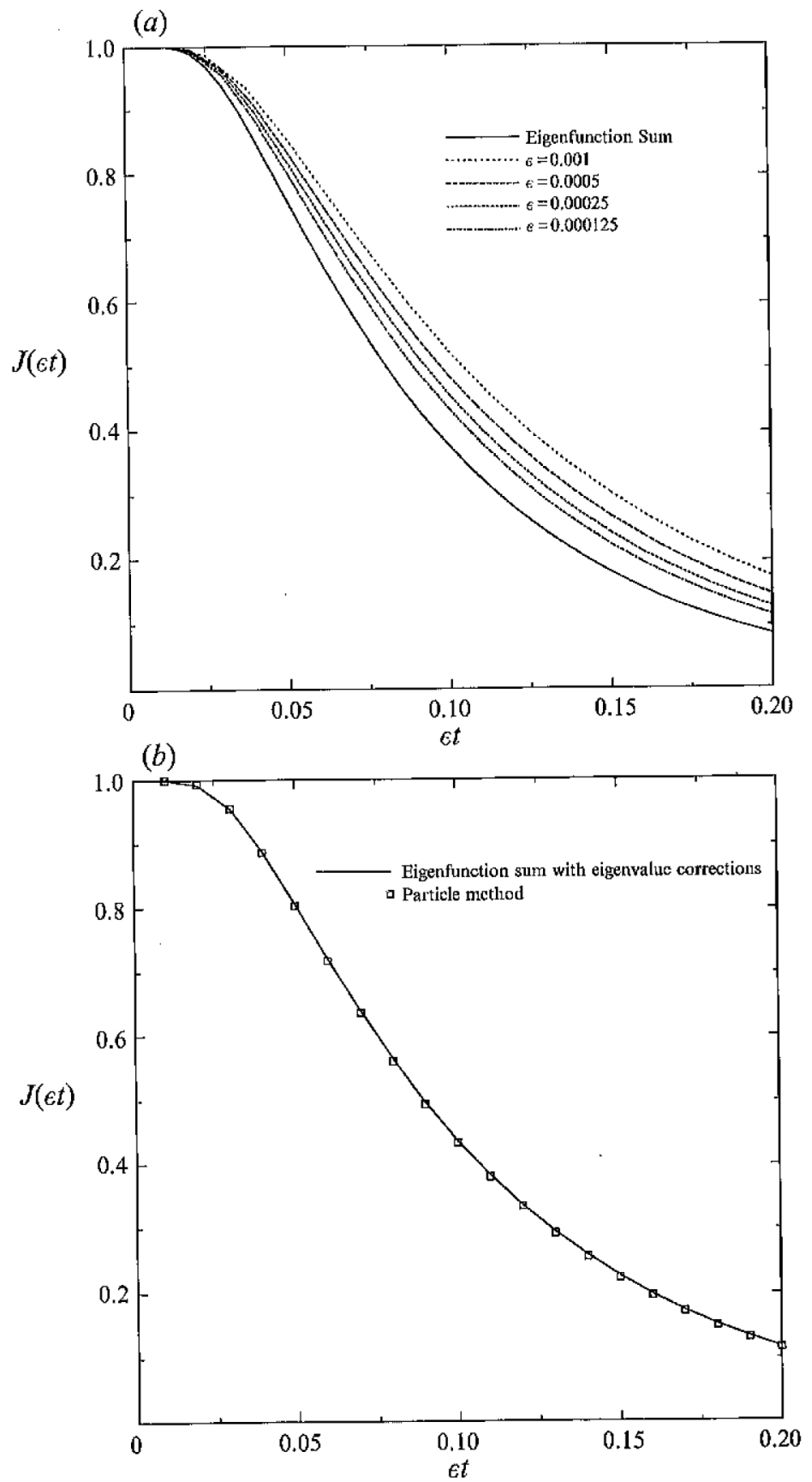

FIGURE 6. Integrated concentration vs. time. (a) Leading-order eigenfunction expansion and numerical solution. The integrated concentration within the dipole decays on an $O(P e)$ timescale $e t$. The convergence of the numerical results to the eigenfunction solution with decreasing $\epsilon$ is slow owing to large correction terms $\left(O\left(\varepsilon^{\frac{1}{2}}\right)\right)$ neglected in the decay rates. $(b)$ Eigenfunction expansion with firstorder correction. By including the order $\epsilon^{\frac{1}{2}}$ in the decay rates of the eigenfunction expansion excellent agreement is obtained between the asymptotic and numerical results, shown here for $c=0.000125$. 


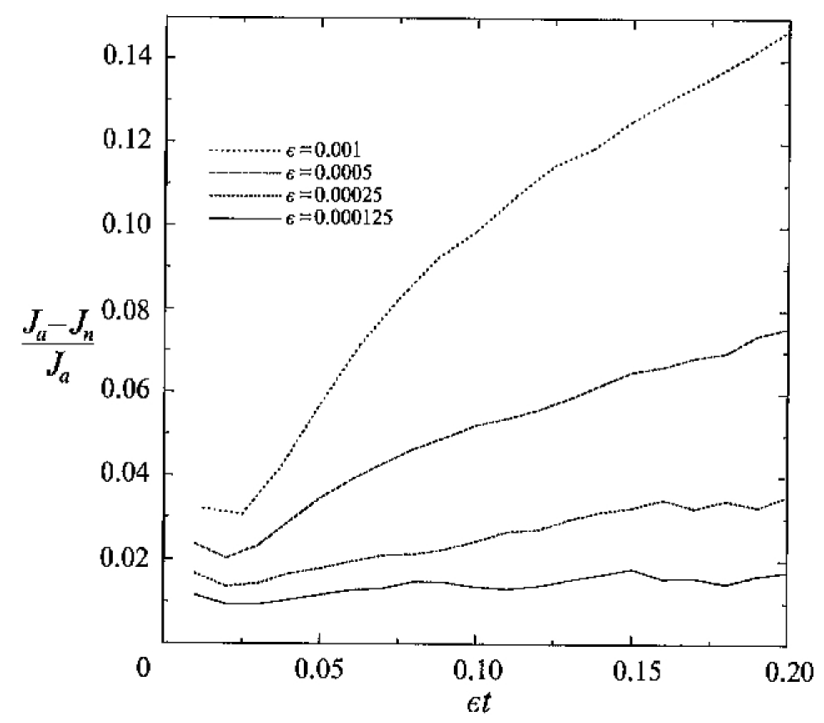

FIGURE 7. Relative error in integrated concentration $v s$. time. The relative error between the eigenfunction solution with $O\left(\sigma^{\frac{1}{2}}\right)$ corrections $J_{a}$, and the numerical solution $J_{n}$, is shown; note that the error decreases approximately proportionally to $\epsilon$. This validates the first two orders of the asymptotic theory. The variation in the curves is due to the probabilistic error of the particle method.

The integrated concentration within the dipole at time $t$ is given by

$$
J(t)=\int_{\mathscr{D}} C(\psi, t) \mathrm{d} A=4 \pi \sum_{n=0}^{\infty} d_{n} \mathrm{e}^{\lambda_{n}^{s} c t}\left\langle 1, \phi_{n}^{s}\right\rangle,
$$

where the integration domain $\mathscr{D}$ is the interior of the Lamb dipole. The coefficients $d_{n}$ in the eigenfunction expansion (5.4) for initial condition (5.14) are

$$
d_{n}=\frac{1}{4 \pi} \frac{\phi_{n}^{s}\left(\psi_{m}\right)}{\left\langle\phi_{n}^{s}, \phi_{n}^{s}\right\rangle}
$$

Figure 6(a) is a comparison of the integrated concentration within the dipole, $J$, computed by (5.15) and a particle method simulation. The solid curve is the eigenfunction solution in the limit $\epsilon \rightarrow 0$ and the dashed curves are the particle-method results for $\epsilon=0.001,0.0005,0.00025,0.000125$. Each of the numerical simulations is an ensemble average of $3 \times 10^{5}$ particles and the numerical time step $\Delta t$, was refined until the numerical time-stepping error became the same size as the probabilistic error (see Appendix A). The decay of the integrated concentration within the dipole occurs on the slow timescale, $s t$, and the convergence of the particle method to the asymptotic limit, $\epsilon \rightarrow 0$, is slow owing to the large $O\left(\epsilon^{\frac{1}{2}}\right)$ correction to the $n=0$ leading-order symmetric decay rate $(5.13 b)$.

Figure $6(b)$ is a graph of integrated concentration within the dipole, $J$, and incorporates the $O\left(\epsilon^{\frac{1}{b}}\right)$ corrections for the decay rates in the eigenfunction solution. The solid curve is the eigenfunction solution for $\epsilon=0.000125$ and the results of the particle method (squares) exactly overlap with this solution. There are also $O\left(\epsilon^{\frac{1}{2}}\right)$ corrections to the coefficients in (5.15) but the numerical error in the particle method is of the same order as these corrections so we could not obtain a numerical measurement of these quantities for comparative purposes.

Figure 7 is the relative error between the eigenfunction and numerical solutions versus time. The relative error consists of three independent contributions. The sources 


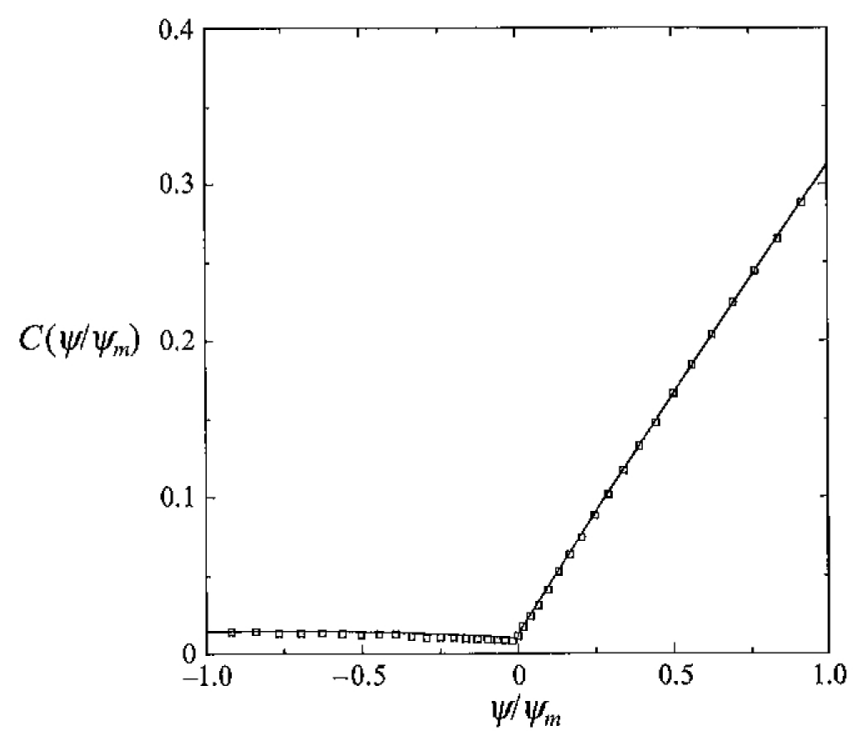

FIGURE 8. Concentration vs. streamfunction for $\epsilon t=0.15$, and $\epsilon=0.000125$. The concentration within the dipole becomes proportional to the slowest decaying eigenmode (i.e. proportional to the streamfunction at leading order). The eigenfunction sum (solid line) agrees well with the data obtained from the particle method (squares).

of error are: the systematic time-stepping error of the numerical method which can be controlled by choosing the time step $\Delta t$ sufficiently small; the probabilistic error of the numerical method due to the random-walk simulation of diffusion which can be controlled by choosing the number of particles sufficiently large; and the asymptotic error in the perturbation theory which can be controlled by choosing the nondimensional diffusion coefficient $\epsilon$ sufficiently small. The time step of the numerical integration was refined until the systematic time-stepping error was buried in the probabilistic noise; thus the chief contribution to the error in figure 7 is from the asymptotic sources. Note that the random fluctuations of the relative error about the mean are due to the statistical error. For a fixed time the relative error in figure 7 is proportional to $\epsilon$; this verifies the asymptotic error estimation. The linear growth of the relative error with time is a result of the higher-order corrections in the decay rates.

Figure 8 is a comparison of the eigenfunction and numerical solutions for the concentration profile within the dipole for $\varepsilon=0.000125$ and $\epsilon t=0.15$. The concentration has been homogenized along streamlines owing to shear dispersion. Figure 8 can be thought of as the concentration profile along a line segment connecting the centres of the dipole lobes. Positive (negative) values of $\psi$ correspond to the upper (lower) half of the dipole. The initial delta function quickly decays to a concentration profile proportional to the streamfunction owing to the more rapid exponential decay of the higher eigenmodes. Then the concentration within the dipole slowly approaches the symmetric eigenmode for large times because of the small difference in the (anti)symmetric decay rates. This can be seen in figure 8 as concentration slowly leaks into the lower half of the dipole from the upper half.

Figure 9 is a comparison of the asymptotic prediction (curves) and numerical measurements (error bars) of the (anti)symmetric eigenmode decay rates, $\lambda_{0}^{a}$, $\left(\lambda_{0}^{s}\right)$ versus the natural logarithm of the Péclet number. The error bars on the numerical measurements are plus or minus one standard deviation and were determined by 


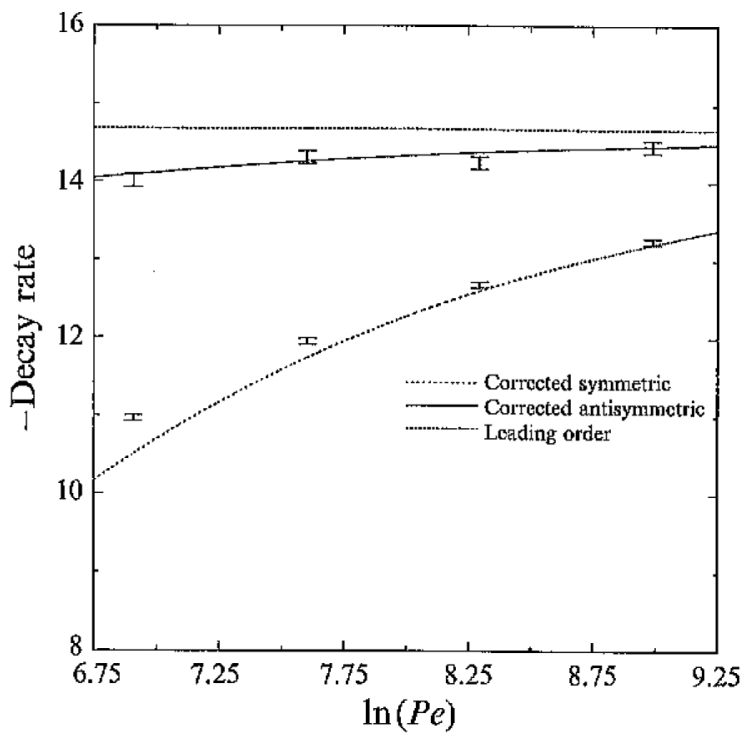

FIGURE 9. Asymptotic decay rate $v$ s. Péclet number. The decay rate for large times as a function of Péclet number is shown for the even and odd eigenfunctions. The dotted line represents the leadingorder estimate of the decay rate, given by the lowest eigenvalue of the averaged diffusion equation. The curves incorporate the $e^{\frac{1}{2}}$ correction to this decay rate. The data with error bars are the measurements of the decay rate by the particle method. Note that the numerical results converge to the asymptotic theory which slowly approaches the leading-order approximation to the lowest eigenvalue as the Péclet number increases.

measuring decay rates of several realizations. The convergence of the decay rates to the asymptotic limit is evident as well as the large correction term to the symmetric decay rate for the range of Péclet numbers, 1000-8000, studied.

\section{Discussion}

The transport of a passive scalar by a Lamb dipole in the limit of large Péclet number has been studied in this paper. It has been shown that an arbitrary initial concentration within the dipole is first averaged on closed streamlines by shear-augmented dispersion on a timescale of order $P e^{\frac{1}{3}}$. Scalar is then transported across the streamlines, on the diffusive timescale, $P e$. A one-dimensional diffusion equation for the mean concentration on streamlines is derived in $\S 3$. At the edge and along the centreline of the dipole a boundary layer of width $P e^{-\frac{1}{2}}$ forms; scalar entering this boundary layer is rapidly expelled and the concentration drops nearly to zero in the boundary layer. The boundary-layer analysis in $\S 4$ allows the derivation of an effective boundary condition for the averaged diffusion equation.

In $\S 5$ the asymptotic results are compared to numerical simulations using a split-step particle method. Excellent agreement is obtained in the large-Péclet-number limit, verifying the efficacy of both the asymptotic and numerical methods. The concentration within the dipole relaxes to a superposition of the lowest two eigenmodes: one symmetric, one antisymmetric and for which the concentration is proportional to the streamfunction. The corresponding eigenvalue determines the rate of decay of concentration within the dipole.

The methodology in this paper extended previous work in two ways. First, an averaging method is introduced that allows us to capture both the initial 
homogenization along streamlines and the slower diffusion across streamlines. We have argued that this process will occur in any region of closed streamlines with nonzero shear. Secondly, we have shown how to apply boundary-layer methods to understand the decay of scalar in an isolated region of closed streamlines; this is an important phenomena for understanding the transport properties of coherent structures, like dipoles.

The decay of the lowest antisymmetric eigenmode is qualitatively a good description of the viscous decay of the Lamb dipole. If the Lamb-dipole velocity field is taken as an initial condition of the full Navier-Stokes equations, the advection-diffusion of vorticity will parallel the advection-diffusion of a passive scalar with the Reynolds number playing the role of the Péclet number. Initially, the vorticity is constant along streamlines and is proportional to the streamfunction (i.e. the vorticity initial condition is in the lowest antisymmetric eigenstate); as noted by Batchelor (1967, p. 534-537) this will lead to a decay of the vorticity at a rate proportional to the lowest eigenvalue. However, this assumption ignores the formation of the boundary layer; in fact, the decaying solution suggested by Batchelor does not conserve vorticity at the edge of the dipole. What is clear from this analysis is that a viscous boundary layer forms at the edge of the dipole, which sweeps vorticity to the rear stagnation point and into the exterior flow. The analysis above clearly demonstrates that this process occurs on an $O(R e)$ timescale. Unfortunately the analysis here can only be used to analyse this behaviour qualitatively because the vorticity in the boundary-layer contribution to the velocity field is $O\left(R e^{-1}\right)$ which distorts the dipole on the same timescale as it decays. The analysis of this slowly decaying dipole is a goal of our future study.

The authors would like to thank Seth Lichter for leading us to this problem and his continuing support of this research. We would like to thank Bruce Bayly, Brian Conlon, Isaac Klapper, Lou Rossi and Jack Xin for helpful discussions and Edgar Knobloch and George Papanicolaou for sharing preprints of their research. This work has been supported by NSF grant CTS-9206828. One of us (A.J.B) would like to thank P.J. and D.J. (Porcupine and Avid) for their continuing support.

\section{Appendix A. Split-step particle method for advection-diffusion equations}

In this Appendix we analyse the order of error of a split-step particle method used to simulate the advection-diffusion equation. The basic idea of a particle method is to exploit the well-known connection between a random walk of a particle and its probability distribution governed by a related diffusion equation (cf. Ghoniem \& Sherman 1985; Klapper 1992).

Our analysis of the numerical scheme is directed differently than much of the literature; we study the error in the approximation of the kernel of the advection-diffusion equation at each time step. This error estimate is then used to analyse the error for integration over a fixed time interval. Previous investigators have concentrated on the error in the trajectory of a single particle governed by a stochastic differential equation (Chang 1987); here we concentrate on the error in the evolution of a distribution of particles. In addition, it is assumed that the primary measurements will be of concentration integrated over a region (e.g. the interior of the dipole). As such, the smoothness of the distribution is relatively unimportant; for reconstructing concentration fields gradient methods are probably more appropriate (Ghoniem \& Sherman 1985; Fogelson 1992).

An additional problem is that the diffusion kernel has sharp gradients, which might 
lead to large errors. However, the method of kernel expansion allows us to examine the form of the error and to show that for the relevant quantities measured here, the error is second-order in time.

As the advection-diffusion problem (3.1) is linear and time independent, it lends itself to a Green's function solution. If a kernel, $K\left(x, x^{\prime}, t\right)$ is defined as the solution to

$$
K_{t}+\boldsymbol{u} \cdot \boldsymbol{\nabla} K=\epsilon \nabla^{2} K+\delta\left(\boldsymbol{x}^{\prime}\right) \delta(t),
$$

then the solution can be propagated forward for a time $\tau$ throughout a convolution,

$$
C(x, t+\tau)=\int \mathrm{d} x^{\prime} K\left(x, x^{\prime}, \tau\right) C\left(x^{\prime}, t\right),
$$

where the integral is taken over all space. In the case of vanishing $u$ the kernel becomes the kernel of the $n$-dimensional diffusion equation

$$
K\left(\boldsymbol{x}, \boldsymbol{x}^{\prime}, t\right)=H(\boldsymbol{r}, t)=(4 \pi \epsilon t)^{-n / 2} \mathrm{e}^{-\mathrm{r}^{2} /(\mathbf{1} \epsilon t)}, \quad r=|\boldsymbol{r}|, \quad \boldsymbol{r}=\boldsymbol{x}-\boldsymbol{x}^{\prime} .
$$

At this point several key observations can be made which allow an expansion of the kernel of the solution. First note that if $\boldsymbol{u}$ is constant the advection-diffusion equation (3.1) can be transformed into the heat equation with a Galilean transformation. Second, the kernel of the heat equation, $H$, is spatially exponentially localized. This leads us to believe that the kernel $K$ can be approximated by $H$; in fact, if the velocity is Taylor expanded, the constant leading-order term can be removed by a Galilean transformation and the higher-order terms can be incorporated as a series of small corrections.

The velocity can be Taylor expanded at a point $x_{0}$ :

$$
u(x)=u_{i}(x)=u_{i}^{(0)}+u_{i j}^{(1)} y_{j}+\frac{1}{2} u_{i j k}^{(2)} y_{j} y_{k}+\ldots,
$$

where $y=x-x_{0}$ and

$$
u_{i}^{(0)}=\left.u_{i}\right|_{x=x_{0}}, \quad u_{i j}^{(1)}=\left.\frac{\partial u_{i}}{\partial x_{j}}\right|_{x=x_{0}}, \quad u_{i j k}^{(2)}=\left.\frac{\partial^{2} u_{i}}{\partial x_{j} \partial x_{k}}\right|_{x=x_{0}} .
$$

The kernel $K$ can now be expanded in terms of $H$; at leading order

$$
K\left(\boldsymbol{x}, x_{0}, t\right) \approx H(\zeta, t), \quad \zeta_{i}=y_{i}-u_{i}^{(0)} t .
$$

Higher-order corrections can all be expressed in terms of powers of $t$ and gradients of $H$. Substituting an expansion of this form leads to the conclusion that

$$
K\left(\boldsymbol{x}, \boldsymbol{x}_{0}, t\right)=H+\frac{\dot{1}}{2} t^{2}\left[u_{i j}^{(1)}\left(2 \epsilon \frac{\partial^{2} H}{\partial x_{i} \partial x_{j}}-u_{i}^{(0)} \frac{\partial H}{\partial x_{i}}\right)-u_{i j j}^{(2)} 2 \epsilon \frac{\partial H}{\partial x_{i}}\right]+\mathscr{E},
$$

where the error term is given by

$$
\mathscr{E}=O\left(t^{3}\left(\frac{\partial H}{\partial x_{i}}, \epsilon \frac{\partial^{2} H}{\partial x_{i} \partial x_{j}}, e^{2} \frac{\partial^{3} H}{\partial x_{i} \partial x_{j} \partial x_{k}}\right)\right)
$$

and where $H$ and it gradients are always evaluated at $\zeta, t$.

We now describe a standard split-step numerical method that reproduces this kernel to order $t^{2}$ with an error term of the same form. The basic idea is to split the equation into an advection step

$$
C_{i}+u \cdot \nabla C=0
$$

and a diffusion step

$$
C_{t}=\epsilon \nabla^{2} C \text {. }
$$


We are considering the evolution of an ensemble of particles: each of these steps can be solved easily for a given particle. The advection step can be solved by integrating the characteristic equation

$$
\mathrm{d} x / \mathrm{d} t=u
$$

with a sufficiently high-order scheme (here fourth-order Runge-Kutta), while the diffusion step is implemented by randomly displacing the particle with a probability density given by the heat-equation kernel (A 3); in two dimensions this is easily accomplished with the Box-Muller transformation (cf. Chang 1987; Fogelson 1991).

The numerical scheme used for a time step $h$ is to do a diffusion step for a time $\frac{1}{2} \mathrm{~h}$, an advection step for a time $h$ and then a second diffusion step for a time $\frac{1}{2} \mathrm{~h}$. Again Taylor expanding the kernel corresponding to each of these processes yields (A 7) with an error term of the form (A 8).

To understand the error due to this numerical scheme, the expression for the error, $\mathscr{E}$, is substituted into the convolution (A 2). Integration by parts yields that the error after a time step of length $h$ for an initial condition $\tilde{C}$ is given by

$$
\mathscr{E}(\tilde{C})=O\left(h^{3}\left(\frac{\partial \tilde{C}}{\partial x_{i}}, \epsilon \frac{\partial^{2} \tilde{C}}{\partial x_{i} \partial x_{j}}, \epsilon^{2} \frac{\partial^{3} \tilde{C}}{\partial x_{i} \partial x_{j} \partial x_{k}}\right)\right) .
$$

This result dovetails nicely with the known error results for split-step schemes (Strang 1968); after integration for a time $T$ the error is proportional to $T h^{2}$. The size of the gradient terms in (A 12) also needs to be estimated. Even if the initial condition is nonsmooth, the eventual state of the system will be a smooth function. From the boundary-layer theory herein we expect the solution to have order-unity gradients but with second derivatives that scale like $e^{-\frac{1}{2}}$. From this estimate it follows that the gradients in (A 12) are of order unity, and as such that the error will scale like $T h^{2}$, independent of $\epsilon$.

Particle methods are also subject to a probabilistic error. Suppose that $N$ particles are used and $p$ particles are found in some region. Probability theory says that in the limit of large $N$ this can be thought of as a single measurement from a Gaussian distribution with standard deviation $\sigma N^{\frac{-1}{2}}$, where $\sigma \approx\left[(p / N)(1-p / N)^{\frac{1}{2}}\right.$. In practice, the standard deviation of the measurements here are easily computed and $N$ is chosen sufficiently large that the probabilistic error is comparable to the time-stepping error.

\section{Appendix B. Numerical solution of the boundary-layer integral equation}

This Appendix describes the formulation and numerical solution of the integral equation for the boundary-layer profile at section $O$ of the Lamb dipole (see figure 2). Consider the boundary-layer solution with odd symmetry about centreline $\overline{F R}$. The boundary conditions on the odd boundary-layer solution are given by

$$
\begin{aligned}
& C=0 \text { along the centreline, } \\
& C \rightarrow a \Psi+b \text { as } \Psi \rightarrow \infty, \\
& C \rightarrow 0 \text { as } \Psi \rightarrow-\infty \text { outside the dipole. }
\end{aligned}
$$

Boundary condition (B $1 b$ ) is really a matching condition between the boundary layer and the WKB solution. The constants $a, b$ cannot be imposed arbitrarily because their ratio is fixed as discussed in $\S 4$. Imposing a unit flux $(a=1)$ at $\Psi=\Psi_{b} \gg 1$ determines the constant $b$ through the solution of the boundary-layer equations. The inhomogeneous boundary condition ( $\mathrm{B} 1 b$ ) can be transferred into a forcing term in the 
integral equation by subtracting out the constant-flux part of the concentration. Let $C(\Psi)$ be an arbitrary initial profile at section $O$ satisfying the boundary condition (B 1), $C^{\prime}\left(\Psi^{\prime}\right)$ be the mapping of $C(\Psi)$ to $O^{\prime}$ and $C^{\prime \prime}\left(\Psi^{\prime \prime}\right)$ be the mapping of $C^{\prime}\left(\Psi^{\prime}\right)$ back to $O$ as discussed in $\S 4$. Define

$$
\begin{aligned}
\hat{C}(\Psi) & =C(\Psi)-\Psi \text { at } \quad O, \\
\hat{C}^{\prime}\left(\Psi^{\prime}\right) & =C^{\prime}\left(\Psi^{\prime}\right)-\frac{1}{2} \Psi^{\prime}\left(1+\operatorname{erf}\left(\Psi^{\prime}\right)\right) \text { at } O^{\prime},
\end{aligned}
$$

where $\hat{C}, \hat{C}^{\prime}$ now satisfy a homogeneous boundary condition $\left(\hat{C}_{\Psi^{\prime}}=0\right)$ in the far field inside the dipole at $O$ and $O^{\prime}$. The mappings from $O$ to $O^{\prime}$ and then from $O^{\prime}$ to $O$ are convolution integrals of the concentration with the boundary-layer kernel:

$$
\begin{gathered}
C^{\prime}\left(\Psi^{\prime}\right)=\int_{0}^{\infty} \mathrm{d} \Psi G_{F}\left(\Psi^{\prime}, \Psi\right) C(\Psi)=G_{F}\left(\Psi^{\prime}, \Psi\right) * C\left(\Psi^{\prime}\right) \\
=\hat{G}_{F}\left(\Psi^{\prime}, \Psi\right) * \hat{C}(\Psi)+\hat{G}_{F}\left(\Psi^{\prime}, \Psi\right) * \Psi, \\
C^{\prime \prime}\left(\Psi^{\prime \prime}\right)=\int_{-\infty}^{\infty} \mathrm{d} \Psi^{\prime \prime} G_{R}\left(\Psi^{\prime \prime}, \Psi^{\prime}\right) C^{\prime}\left(\Psi^{\prime}\right)=G_{R}\left(\Psi^{\prime \prime}, \Psi^{\prime}\right) * C^{\prime}\left(\Psi^{\prime}\right) \\
=\hat{G}_{R}\left(\Psi^{\prime \prime}, \Psi^{\prime}\right) * \hat{C}^{\prime}\left(\Psi^{\prime}\right)+\hat{G}_{R}\left(\Psi^{\prime \prime}, \Psi^{\prime}\right) * \frac{1}{2} \Psi^{\prime}\left(1+\operatorname{erf}\left(\Psi^{\prime}\right)\right) .
\end{gathered}
$$

The kernels $\hat{G}_{F}, \hat{G}_{R}$ are constructed from the Green's function $G$ in (4.17) by the method of images so that the concentrations $\hat{C}^{\prime}, \hat{C}^{\prime \prime}$ satisfy the homogeneous boundary conditions

$$
\begin{aligned}
\hat{C}^{\prime \prime}=0 & \text { along the centreline, } \\
\hat{C}_{\Psi,}^{\prime} \hat{C}_{\psi}^{\prime \prime} \rightarrow 0 & \text { as } \Psi \rightarrow \infty, \\
\hat{C}^{\prime} \rightarrow 0 & \text { as } \quad \Psi \rightarrow-\infty \quad \text { outside the dipole. }
\end{aligned}
$$

The kernels are given by

$$
\begin{gathered}
\hat{G}_{F}\left(\Psi^{\prime}, \Psi\right)= \begin{cases}G\left(\Psi^{\prime}, D_{2} ; \Psi, D_{1}\right)-G\left(\Psi^{\prime}, D_{2} ;-\Psi, D_{1}\right) & \text { for } \Psi^{\prime}<\Psi_{c}, \\
G_{g}\left(\Psi^{\prime}, D_{2} ; \Psi, D_{1}\right)+G_{g}\left(\Psi^{\prime}, D_{2} ; 2 \Psi_{b}-\Psi, D_{1}\right) & \text { for } \Psi^{\prime}>\Psi_{c},\end{cases} \\
\hat{G}_{R}\left(\Psi^{\prime \prime}, \Psi^{\prime}\right)= \begin{cases}G\left(\Psi^{\prime \prime}, D_{1} ; \Psi^{\prime}, D_{2}\right) & \text { for } \Psi^{\prime \prime}<\Psi_{c}, \\
G_{g}\left(\Psi^{\prime \prime}, D_{1} ; \Psi^{\prime}, D_{2}\right)+G_{g}\left(\Psi^{\prime \prime}, D_{1} ; 2 \Psi_{b}-\Psi, D_{2}\right) & \text { for } \Psi^{\prime \prime}>\Psi_{c},\end{cases}
\end{gathered}
$$

where $\Psi_{c}=\frac{1}{2} \Psi_{b} ; \Psi_{b} \gg 1$ is introduced as a numerical artifice to make the computational domain finite, $\left[0, \Psi_{b}\right]$. In practice, the value of $\Psi_{b}$ is increased until the boundary-layer solution becomes independent of $\Psi_{b}$. For $\Psi_{b}$ sufficiently large, $\Psi_{c}$ represents the value of $\Psi$ for which the boundary-layer kernel $G$ becomes the familiar diffusion kernel (i.e. $F=1$ for $\Psi>\Psi_{c}$ ). The boundary condition $\hat{C}_{w}=0$ at $\Psi=\Psi_{b}$ is imposed by the method of images. $G$ is specified in (4.17), $G_{g}$ is $G$ with $F=1$ and $D_{1}\left(D_{2}\right)$ are 1.0922 (1.5433). Using (B $2 a$, B $3 b$ ) to substitute for $\hat{C}^{\prime}$ in (B $3 d$ ) gives an equation for $C^{\prime \prime}$ in terms of $\hat{C}$ :

$$
C^{\prime \prime}\left(\Psi^{\prime \prime}\right)=\hat{G}_{R}\left(\Psi^{\prime \prime}, \Psi^{\prime}\right) * \hat{G}_{F}\left(\Psi^{\prime}, \Psi\right) *(\hat{C}(\Psi)+\Psi)
$$

Equation (B 6) is the return map for the concentration at $O$ due to one half of the dipole. The concentration from the other lobe can be incorporated by requiring the total concentration $C_{T}^{\prime \prime}\left(\Psi^{\prime \prime}\right)$ to be an odd function of $\Psi^{\prime \prime}$ :

$$
C_{T}^{\prime \prime}\left(\Psi^{\prime \prime}\right)=C^{\prime \prime}\left(\Psi^{\prime \prime}\right)-C^{\prime \prime}\left(-\Psi^{\prime \prime}\right)
$$


Equations (B 6) and (B 7) along with the periodicity condition, $\hat{C}_{T}^{\prime \prime}=\hat{C}$, gives an integral equation for $\hat{C}(\Psi)$ :

$$
\hat{C}_{T}^{\prime \prime}\left(\Psi^{\prime \prime}\right)=\hat{G}\left(\Psi^{\prime \prime}, \Psi\right) * \hat{C}(\Psi)+I\left(\Psi^{\prime \prime}\right),
$$

where $I, \hat{G}$ are given by

$$
\begin{aligned}
\hat{G}\left(\Psi^{\prime \prime}, \Psi^{\prime}\right) & =\hat{G}_{R}\left(\Psi^{\prime \prime}, \Psi^{\prime}\right) * \hat{G}_{F}\left(\Psi^{\prime \prime}, \Psi\right)-\hat{G}_{R}\left(-\Psi^{\prime \prime}, \Psi^{\prime}\right) * \hat{G}_{F}\left(\Psi^{\prime}, \Psi\right) \\
I\left(\Psi^{\prime \prime}\right) & =\hat{G}\left(\Psi^{\prime \prime}, \Psi\right) * \Psi_{-} \Psi^{\prime \prime}
\end{aligned}
$$

The inhomogeneous term $I\left(\Psi^{\prime \prime}\right)$ is an odd function of $\Psi^{\prime \prime \prime}$ and decays to zero as $\Psi^{\prime \prime \prime} \rightarrow \infty$.

The numerical solution of (B 8) is accomplished by a forward iteration of the integral equation:

$$
\hat{C}_{n+1}\left(\Psi^{\prime \prime}\right)=\hat{G}\left(\Psi^{\prime \prime}, \Psi\right) * \hat{C}_{n}(\Psi)+I\left(\Psi^{\prime \prime}\right) .
$$

Physically, the equation corresponds to a mapping for a diffusion process; thus the eigenvalues of the operator $\hat{G}$ are less than unity and a forward iteration of the equation will converge to the boundary-layer solution. The convolution integrals become matrix multiplications when the integral equation is discretized. Because the kernels are exponentially localized within the boundary layer, the trapezoid rule can be utilized to numerically integrate the convolutions with exponential accuracy.

The even boundary-layer solution is found in an exactly analogous manner. The boundary condition along the centreline, $\overline{F R}$, becomes $C_{\Psi}=0$ and instead of subtracting an odd function of $\Psi$ to homogenize the far-field boundary condition, (B $2 a$ ) is replaced by

$$
\hat{C}(\Psi)=C(\Psi)-\Psi \operatorname{erf}(\Psi) \text { at } O
$$

The boundary-layer solutions are given in figure 4 .

\section{REFERENCES}

Ahlnas, K., Royer, T. C. \& Grorge, T. H. 1987 Multiple dipole eddies in the Alaska Coastal Current detected with Landsat thematic mapper data. J. Geophys. Res. 92, 13041-13047.

BATCHELOR, G. K. 1967 An Introduction to Fhid Dynamics. Cambridge University Press.

Bernard, P. S. 1990 Convective diffusion in two-dimensional incompressible linear flow. SIAM Rev. 32, 660-666.

Burgers, J. M. 1948 A mathematical model illustrating the theory of turbulence. Adv. Appl. Mech. 1, 171-199.

Chang, C. C. 1987 Numerical solution of stochastic differential equations with constant diffusion coefficients. Maths of Comput. 49, 523-542.

Childress, S. 1979 Alpha-effect in flux ropes and sheets. Phys. Earth Planet. Interiors 20, 172-180.

Childress, S. \& Soward, A. M. 1989 Scalar transport and alpha-effect for a family of cat's-eye flows. J. Fluid Mech. 205, 99-133.

Conlon, B. \& Lichrer, S. 1994 Wall jet induced dipole formation: a numerical study. In preparation.

COUdER, Y. \& BASDEvant, C. 1986 Experimental and numerical study of vortex couples in twodimensional flows. J. Fluid Mech. 173, 225-251.

FanNJian, A. \& PapanicolaOU, G, 1994 Convection enhanced diffusion for periodic flows. SIAM J. Appl. Maths (to appear).

FogrLSON, A. L. 1992 Particle-method solution of two-dimensional convection-diffusion equations. J. Comput. Phys, 100,1-16.

FowLer, A. C. 1985 Secular cooling in convection. Stud. Appl. Maths 72, 161-171.

Ghoniem, A. F. \& Sherman, F. S. 1985 Grid-free simulation of diffusion using random walk methods. J. Comput. Phys. 61, 1-37. 
HARPER, J. F, 1963 On boundary layers in two-dimensional flow with velocity. J. Fluid Mech. 17, 141-153.

HEIJST, G. J. F. VAN \& FLón, J. B. 1989 Dipole formation and collisions in a stratified fluid, Nature $340,212-215$.

KLAPPER, I. 1992 Shadowing and the role of small diffusivity in the chaotic advection of scalars. Phys. Fluids A 4, 861-864.

KNOBLOCH, E. \& Merrypield, W. J. 1992 Enhancement of diffusive transport in oscillatory flows. Astrophys. J. 401, 196-205.

MCCARTY, P. \& Horsthfimke, W, 1988 Effective diffusion coefficient for steady two-dimensional flow. Phys. Rev. A 37, 2112-2117.

MCWILLIAMs,. J. C. 1984 The emergence of isolated coherent vortices in turbulent flow. $J$. Fluid Mech. 146, 21-43.

NEwTON, P. K. \& Meiburg, E. 1991 Particle dynamics in at viscously decaying cat's eye: The effect of finite Schmidt numbers. Phys. Fluids A 3, 1068-1072.

Perkins, F. W. \& Zweibel, E. G. 1987 A high magnetic Reynolds number dynamo. Phys. Fluids 30, $1079-1084$.

RHINES, P. B. \& Young, W. R. 1983 How rapidly is a passive scalar mixed within closed streamlines? J. Fluid Mech. 133, 133-145.

Rosennluth, M. N., Berk, H. L., Doxas, I. \& Horton, W. 1987 Effective diffusion in laminar convective flows. Phys. Fluids 30, 2636-2647.

Saffman, P. G. 1992 Vortex Dynamics. Cambridge University Press.

Shraiman, B. I. 1987 Diffusive transport in a Rayleigh-Bénard convection cell. Phys. Rev. A 36, 261-267.

Soward, A. M. 1987 Fast dynamo action in a steady flow. J. Fluid Mech. 180, 267-295.

STRANG, G. 1968 On the construction and comparison of different schemes. SIAM J. Numer. Anal. 5, 506-517.

Swaters, G. E. 1988 Viscous modulation of the Lamb dipole vortex. Phys. Fluids 31, 2745-2747.

TAYLOR, G. I. 1953 Dispersion of soluble matter in solvent flowing slowly through a tube. Proc. $R$. Soc. Lond. A 219, 186-203.

Young, W. R. \& Jones, S. 1991 Shear dispersion, Phys. Fluids A 3, 1087-1101.

Young, W., Pumir, A. \& Pomeau, Y. 1989 Anomalous diffusion of tracer in convection rolls. Phys. Fluids A 1, 462-469. 